\title{
Continuous trigonometric collocation polynomial approximations with geometric and superconvergence analysis for efficiently solving semi-linear highly oscillatory hyperbolic systems
}

\section{Changying $\mathrm{Liu}^{1} \cdot$ Xinyuan $\mathrm{Wu}^{2,3}$}

Received: 20 October 2019 / Revised: 2 October 2020 / Accepted: 15 December 2020 /

Published online: 1 February 2021

(c) The Author(s) 2021

\begin{abstract}
In this paper, based on the continuous collocation polynomial approximations, we derive and analyse a class of trigonometric collocation integrators for solving the highly oscillatory hyperbolic system. The symmetry, convergence and energy conservation of the continuous collocation polynomial approximations are rigorously analysed in details. Moreover, we also proved that the continuous collocation polynomial approximations could achieve at superconvergence by choosing suitable collocation points. Numerical experiments verify our theoretical analysis results, and demonstrate the remarkable superiority in comparison with the traditional temporal integration methods in the literature.
\end{abstract}

Keywords Hyperbolic systems · Structure-preserving integrators ·

Superconvergence $\cdot$ Trigonometric polynomial approximations $\cdot$ Highly oscillatory systems

Mathematics Subject Classification 65L07 - 65L20 - 65L70 · 65M12 - 65M15 . $65 \mathrm{M} 22$

Changying Liu

chyliu88@gmail.com

Xinyuan $\mathrm{Wu}$

xywu@nju.edu.cn

1 School of Mathematics and Statistics, Nanjing University of Information Science and Technology, Nanjing 210044, People's Republic of China

2 Department of Mathematics, Nanjing University, Nanjing 210093, People's Republic of China

3 School of Mathematical Sciences, Qufu Normal University, Qufu 273165,

People's Republic of China 


\section{Introduction}

The recent growth in extended Runge-Kutta-Nyström (ERKN) methods (see, e.g. [12, $16,33-35,38-40,42])$ for second-order multi-frequency highly oscillatory systems and the collocation methods for stiff differential equations (see, e.g. $[16,18,32,36]$ ) has let to the development of numerical integrators which systematically incorporate qualitative information transmitted from the underlying problem into their structure. In this paper, we focus on the design and analysis of temporal approximations through continuous trigonometric collocation polynomials for the semi-linear highly oscillatory hyperbolic system:

$$
\left\{\begin{array}{l}
\ddot{u}(t)+A u(t)=f(t, u(t)), \quad t \in\left[t_{0}, T\right], \\
u\left(t_{0}\right)=\varphi, \quad \dot{u}\left(t_{0}\right)=\psi,
\end{array}\right.
$$

where $A \in \mathbb{R}^{d \times d}$ is supposed to be a symmetric positive definite or skew-Hermitian time-invariant matrix with the decomposition $A=\Omega^{2}, f:\left[t_{0}, T\right] \times \mathbb{R}^{d} \rightarrow \mathbb{R}^{d}$ is a nonlinear function, $\varphi$ and $\psi$ are the given initial values of the position $u(t)$ and velocity $\dot{u}(t)$, respectively. This hyperbolic system plays an important role in a wide variety of practical application areas in science and engineering, including nonlinear optics, solid state physics and quantum field theory (see, e.g. [1, 5, 11, 29]). As is known, with suitable spatial discretisation strategies, such as the finite difference method, the finite element method and the spectral method (see, e.g. [3, 14, 17, 20, $21,26-28,30,31])$, the semi-linear wave equations can be converted into the highly oscillatory system (1.1). Therefore, it will be significant to further develop new approaches for efficiently solving the semi-linear highly oscillatory system.

In recent works, based on the operator spectrum theory and the Duhamel's principle, the evolutionary partial differential equations (PDEs) have been formulated as abstract ordinary differential equations (ODEs) in suitable Banach spaces (see, e.g. [4, 19, 22-24]). Therefore, in this paper, we will consider both wave equations in highdimensions and second-order highly oscillatory ordinary differential equations as an semi-linear hyperbolic system (1.1) in a suitable Banach space $(\mathcal{X},\|\cdot\|)$, and present a new temporal integration strategy and related theoretical analyses. If the nonlinear function $f:\left[t_{0}, T\right] \times \mathcal{X} \rightarrow \mathcal{X}$ is continuous, then the solution of the semi-linear hyperbolic system (1.1) and its derivative satisfy the following variation-of-constants formula (see, e.g. [4, 22-24, 38, 40, 42])

$$
\left\{\begin{aligned}
u(t)= & \phi_{0}\left(\left(t-t_{0}\right)^{2} A\right) u\left(t_{0}\right)+\left(t-t_{0}\right) \phi_{1}\left(\left(t-t_{0}\right)^{2} A\right) \dot{u}\left(t_{0}\right) \\
& +\int_{t_{0}}^{t}(t-z) \phi_{1}\left((t-z)^{2} A\right) f(z, u(z)) \mathrm{d} z \\
\dot{u}(t)= & -\left(t-t_{0}\right) A \phi_{1}\left(\left(t-t_{0}\right)^{2} A\right) u\left(t_{0}\right)+\phi_{0}\left(\left(t-t_{0}\right)^{2} A\right) \dot{u}\left(t_{0}\right) \\
& +\int_{t_{0}}^{t} \phi_{0}\left((t-z)^{2} A\right) f(z, u(z)) \mathrm{d} z .
\end{aligned}\right.
$$

Since $A=\Omega^{2}$, the operators $\phi_{0}\left(\left(t-t_{0}\right)^{2} A\right)$ and $\phi_{1}\left(\left(t-t_{0}\right)^{2} A\right)$ are defined as

$$
\phi_{0}\left(\left(t-t_{0}\right)^{2} A\right)=\cos \left(\left(t-t_{0}\right) \Omega\right) \quad \text { and } \quad \phi_{1}\left(\left(t-t_{0}\right)^{2} A\right)=\operatorname{sinc}\left(\left(t-t_{0}\right) \Omega\right) \text {, }
$$


respectively. Furthermore, for any $z \in[0,1]$, the solution of the hyperbolic system (1.1) and its derivative at time $t=t_{n}+z h \in\left[t_{n}, t_{n}+h\right] \subseteq\left[t_{0}, T\right], n=0,1,2, \ldots$ can be expressed in

$$
\left\{\begin{aligned}
u\left(t_{n}+z h\right)= & \phi_{0}\left(z^{2} V\right) u\left(t_{n}\right)+z h \phi_{1}\left(z^{2} V\right) \dot{u}\left(t_{n}\right) \\
& +h^{2} \int_{0}^{z}(z-\tau) \phi_{1}\left((z-\tau)^{2} V\right) g\left(t_{n}+\tau h\right) \mathrm{d} \tau \\
\dot{u}\left(t_{n}+z h\right)= & -z h A \phi_{1}\left(z^{2} V\right) u\left(t_{n}\right)+\phi_{0}\left(z^{2} V\right) \dot{u}\left(t_{n}\right) \\
& +h \int_{0}^{z} \phi_{0}\left((z-\tau)^{2} V\right) g\left(t_{n}+\tau h\right) \mathrm{d} \tau
\end{aligned}\right.
$$

where $V=h^{2} A$ and $g\left(t_{n}+\tau h\right)=f\left(t_{n}+\tau h, u\left(t_{n}+\tau h\right)\right)$. A considerable amount of attention has been paid to the variation-of-constants formula (1.2) or (1.4) to design effective and efficient numerical integrators for solving the highly oscillatory system (1.1). Approximating the nonlinear integrals appearing in formula (1.2) or (1.4) by suitable numerical quadrature formulas, a variety of numerical integrators have been proposed and analysed, including the Gautschi-type method of order two (see, e.g. $[8-10,12,15])$, the adapted Runge-Kutta-Nyström (ARKN) method (see, e.g. $[7,41]$ ), the extended Runge-Kutta-Nyström (ERKN) method (see, e.g. [6, 25, 34, 35, 38, 40, 42]), the arbitrarily high-order Birkhoff-Hermite (BH) method [22], and the trigonometric Fourier collocation (TFC) method [32, 36]. The integrators derived by using the variation-of-constants formula (1.2) or (1.4), such as Gautschitype method, ARKN method, ERKN method, BH method and TFC method etc., are also termed trigonometric integrators. Therefore, the trigonometric integrators can exactly integrate the multi-frequency unperturbed highly oscillatory system $\ddot{u}(t)+A u(t)=0$ associated with (1.1). In other words, the trigonometric integrators can preserve the oscillatory structure [39] of the hyperbolic system (1.1), since the highly oscillatory behaviour of (1.1) is brought by the linear term $A u$.

Moreover, it is well known that the collocation integrators not only provide a discrete set of approximations, but also a continuous approximation to the original solution (see [16]). By choosing suitable collocation nodes, the collocation methods could achieve a highest convergence order. In the recent research work (see $[32,36])$, Wang et al. took advantage of the shifted Legendre polynomials and the Lagrange polynomials to derive two kinds of trigonometric collocation methods for solving the system (1.1). However, these two kinds of trigonometric collocation methods are discrete approximations to original solutions. In addition, the local error bounds and the long-term convergence analysis are insufficient. Therefore, in this paper, using the continuous polynomial approximation, we will focus on combing the superior performance of the collocation integrators with the trigonometric integrators to design the continuous trigonometric collocation polynomial approximation for solving the highly oscillatory hyperbolic system (1.1), which could continuously approximate the original solution and preserve the oscillatory structure of the underlying system. Furthermore, the structure-preserving behaviour, single step error bounds, the long-term convergence and the superconvergence of the continuous trigonometric collocation polynomial approximations will be rigourously investigated in this work.

This paper is organised as follows. In Sect. 2, we formulate the trigonometric collocation integrators based on the continuous polynomial approximation, and 
investigate the symmetry of the trigonometric collocation integrator. Section 3 is concerned with the rigorous analysis of single step error bounds, the long-term convergence, and the superconvergence of the continuous trigonometric collocation polynomial approximations. By choosing suitable collocation nodes, the proposed trigonometric collocation integrators can achieve a highest order of convergence. Furthermore, the long-term energy conservation is also analysed in this section. In Sect. 4, we display preliminary numerical results which demonstrate the advantages and efficiency of our new algorithms in comparison with the existing numerical methods in the literature. The last section is devoted to brief conclusions.

\section{Formulation of the continuous collocation polynomial approximation}

An essential feature of the collocation integrator is that we not only obtain a discrete numerical solution, but also a continuous polynomial approximation to the solution of the original system (Hairer et. al. [16]). In this section, using continuous collocation polynomial approximations, we will derive a class of trigonometric collocation integrators for solving the highly oscillatory hyperbolic system (1.1) and analyse its symmetry. Our analysis for the collocation integrator will be based on the abstract formulation of the hyperbolic equation (1.1) as an evolution equation in a $\operatorname{Banach} \operatorname{space}(\mathcal{X},\|\cdot\|)$.

Definition 2.1 Let $0 \leq c_{1}<c_{2}<\cdots<c_{s} \leq 1$ be distinct nodes. The continuous collocation polynomial approximation is to find a polynomial $y(t)$ of degree $s+1$ in the Banach space $(\mathcal{X},\|\cdot\|)$ such that,

$$
\left\{\begin{array}{l}
y\left(t_{n}\right)=u^{n}, \quad \dot{y}\left(t_{n}\right)=\dot{u}^{n} \\
\ddot{y}\left(t_{n}+c_{i} h\right)=-A y\left(t_{n}+c_{i} h\right)+f\left(t_{n}+c_{i} h, y\left(t_{n}+c_{i} h\right)\right), \\
i=1,2, \ldots, s
\end{array}\right.
$$

Then the numerical solution of the hyperbolic system (1.1) is defined by $u^{n+1}=y\left(t_{n}+h\right)$ and $\dot{u}^{n+1}=\dot{y}\left(t_{n}+h\right)$.

At first glance, it is difficult to seek such a polynomial in practice. Fortunately, following the Lagrange interpolation formula, the continuous collocation polynomial $y(t)$ satisfies

$$
\ddot{y}\left(t_{n}+z h\right)=-A y\left(t_{n}+z h\right)+\sum_{i=1}^{s} l_{i}(z) f\left(t_{n}+c_{i} h, y\left(t_{n}+c_{i} h\right)\right) .
$$

Here and in what follows, we always assume that $z$ is in $[0,1]$. Applying the variation-of-constants formula to (2.2), we obtain 


$$
\left\{\begin{aligned}
y\left(t_{n}+z h\right)= & \phi_{0}\left(z^{2} V\right) y\left(t_{n}\right)+z h \phi_{1}\left(z^{2} V\right) \dot{y}\left(t_{n}\right) \\
& +h^{2} \sum_{i=1}^{s} \int_{0}^{z}(z-\tau) \phi_{1}\left((z-\tau)^{2} V\right) l_{i}(\tau) \mathrm{d} \tau f\left(t_{n}+c_{i} h, y\left(t_{n}+c_{i} h\right)\right), \\
\dot{y}\left(t_{n}+z h\right)= & -z h A \phi_{1}\left(z^{2} V\right) y\left(t_{n}\right)+\phi_{0}\left(z^{2} V\right) \dot{y}\left(t_{n}\right) \\
& +h \sum_{i=1}^{s} \int_{0}^{z} \phi_{0}\left((z-\tau)^{2} V\right) l_{i}(\tau) \mathrm{d} \tau f\left(t_{n}+c_{i} h, y\left(t_{n}+c_{i} h\right)\right) .
\end{aligned}\right.
$$

This seems like that the numerical solution of the continuous collocation polynomial approximations determined by Definition 2.1 could coincide with a class of ERKN integrators (see, e.g.[38, 40, 42]). The details will be confirmed in the following theorem.

Theorem 2.1 The continuous collocation polynomial approximations determined by Definition 2.1 are equivalent to the ERKN methods of the form:

$$
\left\{\begin{array}{l}
u^{n+1}=\phi_{0}(V) u^{n}+h \phi_{1}(V) \dot{u}^{n}+h^{2} \sum_{i=1}^{s} b_{i}(V) f\left(t_{n}+c_{i} h, U^{n i}\right), \\
\dot{u}^{n+1}=-h A \phi_{1}(V) u^{n}+\phi_{0}(V) \dot{u}^{n}+h \sum_{i=1}^{s} \bar{b}_{i}(V) f\left(t_{n}+c_{i} h, U^{n i}\right), \\
U^{n i}=\phi_{0}\left(c_{i}^{2} V\right) u^{n}+c_{i} h \phi_{1}\left(c_{i}^{2} V\right) \dot{u}^{n}+h^{2} \sum_{j=1}^{s} a_{i j}(V) f\left(t_{n}+c_{j} h, U^{n j}\right), \\
\quad i=1,2, \ldots, s
\end{array}\right.
$$

with the weights

$$
\begin{aligned}
& b_{i}(V)=\int_{0}^{1}(1-z) \phi_{1}\left((1-z)^{2} V\right) l_{i}(z) \mathrm{d} z, \\
& \bar{b}_{i}(V)=\int_{0}^{1} \phi_{0}\left((1-z)^{2} V\right) l_{i}(z) \mathrm{d} z
\end{aligned}
$$

and

$$
a_{i j}(V)=\int_{0}^{c_{i}}\left(c_{i}-z\right) \phi_{1}\left(\left(c_{i}-z\right)^{2} V\right) l_{j}(z) \mathrm{d} z,
$$

for $i, j=1,2, \ldots, s$, and $l_{i}(z)$ is the ith Lagrange basis polynomial.

Proof It straightforwardly follows from the formula (2.3) that

$$
\left\{\begin{array}{l}
y\left(t_{n}+h\right)=\phi_{0}(V) y\left(t_{n}\right)+h \phi_{1}(V) \dot{y}\left(t_{n}\right)+h^{2} \sum_{i=1}^{s} b_{i}(V) f\left(t_{n}+c_{i} h, y\left(t_{n}+c_{i} h\right)\right), \\
\dot{y}\left(t_{n}+h\right)=-h A \phi_{1}(V) y\left(t_{n}\right)+\phi_{0}(V) \dot{y}\left(t_{n}\right)+h \sum_{i=1}^{s} \bar{b}_{i}(V) f\left(t_{n}+c_{i} h, y\left(t_{n}+c_{i} h\right)\right), \\
y\left(t_{n}+c_{i} h\right)=\phi_{0}\left(c_{i}^{2} V\right) y\left(t_{n}\right)+c_{i} h \phi_{1}\left(c_{i}^{2} V\right) \dot{y}\left(t_{n}\right)+h^{2} \sum_{j=1}^{s} a_{i j}(V) f\left(t_{n}+c_{j} h, y\left(t_{n}+c_{j} h\right)\right), \\
\quad i=1,2, \ldots, s .
\end{array}\right.
$$


where the weights $b_{i}(v), \bar{b}_{i}(v)$ and $a_{i j}(V)$ are given by (2.5) and (2.6), respectively. The statement of the theorem is proved.

Hairer et al. [16] have pointed out that all second order differential equation $\ddot{u}=\tilde{f}(u)$ written as $\dot{u}=v$ and $\dot{v}=\tilde{f}(u)$ are time reversible. The system (1.1) could be expressed as

$$
\dot{u}(t)=v(t) \quad \text { and } \quad \dot{v}(t)=-A u(t)+f(u(t)):=\tilde{f}(u(t)) .
$$

Therefore, the system (1.1) is clearly time reversible. Moreover, as is known, symmetric methods have excellent long-time behavior when solving reversible differential systems (see Hairer et al. [16]). The design and analysis of the symmetric integrators will be significant in the spirit of geometric integration. Thus, a most welcome feature of the continuous collocation polynomial approaches (2.1) is that it preserves the temporal symmetry by choosing suitable collocation nodes. We are now in a position to prove the symmetry of the trigonometric collocation integrators determined by Definition 2.1.

Theorem 2.2 If the collocation nodes $c_{1}, \ldots, c_{s}$ are symmetric nodes, i.e., $c_{i}+c_{s-i+1}=1$ for $i=1,2, \ldots, s$, then the numerical solutions of the continuous collocation polynomial approximations (2.1) or the extended Runge-Kutta-Nyström integrators (2.4) are symmetric.

Proof We only need to prove that the ERKN methods determined by (2.4) are symmetric. Exchanging $u^{n+1} \leftrightarrow u^{n}, \dot{u}^{n+1} \leftrightarrow \dot{u}^{n}$ and replacing $h$ by $-h$ yields

$$
\begin{gathered}
u^{n}=\phi_{0}(V) u^{n+1}-h \phi_{1}(V) \dot{u}^{n+1}+h^{2} \sum_{i=1}^{s} b_{i}(V) f\left(t_{n+1}-c_{i} h, U_{*}^{n i}\right), \\
\dot{u}^{n}=h A \phi_{1}(V) u^{n+1}+\phi_{0}(V) \dot{u}^{n+1}-h \sum_{i=1}^{s} \bar{b}_{i}(V) f\left(t_{n+1}-c_{i} h, U_{*}^{n i}\right), \\
U_{*}^{n i}=\phi_{0}\left(c_{i}^{2} V\right) u^{n+1}-c_{i} h \phi_{1}\left(c_{i}^{2} V\right) \dot{u}^{n+1} \\
+h^{2} \sum_{j=1}^{s} a_{i j}(V) f\left(t_{n+1}-c_{j} h, U_{*}^{n j}\right), \quad i=1,2, \ldots, s .
\end{gathered}
$$

It follows from combining $\phi_{0}(V) \times\left(2.8+h \phi_{1}(V) \times(2.9)\right.$ that

$$
\begin{aligned}
u^{n+1}=\phi_{0} & (V) u^{n}+h \phi_{1}(V) \dot{u}^{n}+h^{2} \sum_{i=1}^{s}\left(\bar{b}_{i}(V) \phi_{1}(V)-b_{i}(V) \phi_{0}(V)\right) \\
& \times f\left(t_{n}+c_{s-i+1} h, U_{*}^{n i}\right) .
\end{aligned}
$$

Similarly, the transformation $-h A \phi_{1}(V) \times(2.8)+\phi_{0}(V) \times(2.9)$ yields 


$$
\begin{aligned}
\dot{u}^{n+1}=- & h A \phi_{1}(V) u^{n}+\phi_{0}(V) \dot{u}^{n}+h \sum_{i=1}^{s}\left(b_{i}(V) V \phi_{1}(V)+\bar{b}_{i}(V) \phi_{0}(V)\right) \\
& \times f\left(t_{n}+c_{s-i+1} h, U_{*}^{n i}\right) .
\end{aligned}
$$

The symmetric nodes $c_{1}, c_{2}, \ldots, c_{s}$ demonstrate an interesting fact that

$$
l_{i}(1-z)=l_{s-i+1}(z) \text { for } i=1,2, \ldots, s .
$$

Therefore, according to the relations (1.3) and the definition of $b_{i}(V)$ and $\bar{b}_{i}(V)$, it can be confirmed that

$$
\begin{aligned}
\bar{b}_{i}(V) \phi_{1}(V)-b_{i}(V) \phi_{0}(V)= & \int_{0}^{1}\left(\phi_{0}\left((1-\tau)^{2} V\right)\right. \\
& \left.\times \phi_{1}(V)-(1-\tau) \phi_{1}\left((1-\tau)^{2} V\right) \phi_{0}(V)\right) l_{i}(\tau) \mathrm{d} \tau \\
= & \int_{0}^{1}(\cos ((1-\tau) h \Omega) \operatorname{sinc}(h \Omega)-(1-\tau) \operatorname{sinc}((1-\tau) h \Omega) \\
& \times \cos (h \Omega)) l_{i}(\tau) \mathrm{d} \tau \\
= & \int_{0}^{1} \tau \operatorname{sinc}(\tau h \Omega) l_{i}(\tau) \mathrm{d} \tau=\int_{0}^{1} \tau \phi_{1}\left(\tau^{2} V\right) l_{i}(\tau) \mathrm{d} \tau \\
= & \int_{0}^{1}(1-\tau) \phi_{1}\left((1-\tau)^{2} V\right) l_{i}(1-\tau) \mathrm{d} \tau \\
= & \int_{0}^{1}(1-\tau) \phi_{1}\left((1-\tau)^{2} V\right) l_{s-i+1}(\tau) \mathrm{d} \tau=b_{s-i+1}(V)
\end{aligned}
$$

and

$$
\begin{aligned}
b_{i}(V) V \phi_{1}(V)+\bar{b}_{i}(V) \phi_{0}(V)= & \int_{0}^{1}\left((1-\tau) V \phi_{1}\left((1-\tau)^{2} V\right) \phi_{1}(V)\right. \\
& \left.+\phi_{0}\left((1-\tau)^{2} V\right) \phi_{0}(V)\right) l_{i}(\tau) \mathrm{d} \tau \\
= & \int_{0}^{1}((1-\tau) V \operatorname{sinc}((1-\tau) h \Omega) \operatorname{sinc}(h \Omega) \\
& +\cos ((1-\tau) h \Omega) \cos (h \Omega)) l_{i}(\tau) \mathrm{d} \tau \\
= & \int_{0}^{1} \cos (\tau h \Omega) l_{i}(\tau) \mathrm{d} \tau=\int_{0}^{1} \phi_{0}\left(\tau^{2} V\right) l_{i}(\tau) \mathrm{d} \tau \\
= & \int_{0}^{1} \phi_{0}\left((1-\tau)^{2} V\right) l_{i}(1-\tau) \mathrm{d} \tau \\
= & \int_{0}^{1} \phi_{0}\left((1-\tau)^{2} V\right) l_{s-i+1}(\tau) \mathrm{d} \tau=\bar{b}_{s-i+1}(V) .
\end{aligned}
$$

The Eqs. (2.11) and (2.12) can be simplified as 


$$
u^{n+1}=\phi_{0}(V) u^{n}+h \phi_{1}(V) \dot{u}^{n}+h^{2} \sum_{i=1}^{s} b_{s-i+1}(V) f\left(t_{n}+c_{s-i+1} h, U_{*}^{n i}\right),
$$

and

$$
\dot{u}^{n+1}=-h A \phi_{1}(V) u^{n}+\phi_{0}(V) \dot{u}^{n}+h \sum_{i=1}^{s} \bar{b}_{s-i+1}(V) f\left(t_{n}+c_{s-i+1} h, U_{*}^{n i}\right) .
$$

Inserting the Eqs. (2.13) and (2.14) into (2.10) with a careful calculation gives

$$
\begin{aligned}
U_{*}^{n i}= & \left(\phi_{0}\left(c_{i}^{2} V\right) \phi_{0}(V)+c_{i} V \phi_{1}\left(c_{i}^{2} V\right) \phi_{1}(V)\right) u^{n} \\
& +h\left(\phi_{0}\left(c_{i}^{2} V\right) \phi_{1}(V)-c_{i} \phi_{1}\left(c_{i}^{2} V\right) \phi_{0}(V)\right) \dot{u}^{n} \\
& +h^{2} \sum_{j=1}^{s}\left(\phi_{0}\left(c_{i}^{2} V\right) b_{s-j+1}(V)-c_{i} \phi_{1}\left(c_{i}^{2} V\right) \bar{b}_{s-j+1}(V)+a_{i j}(V)\right) \\
& \times f\left(t_{n}+c_{s-j+1} h, U_{*}^{n i}\right) .
\end{aligned}
$$

It then can be obtained after a series of complicated calculations that

$$
\begin{aligned}
& \phi_{0}\left(c_{i}^{2} V\right) \phi_{0}(V)+c_{i} V \phi_{1}\left(c_{i}^{2} V\right) \phi_{1}(V)=\phi_{0}\left(\left(1-c_{i}\right)^{2} V\right), \\
& \phi_{0}\left(c_{i}^{2} V\right) \phi_{1}(V)-c_{i} \phi_{1}\left(c_{i}^{2} V\right) \phi_{0}(V)=\left(1-c_{i}\right) \phi_{1}\left(\left(1-c_{i}\right)^{2} V\right),
\end{aligned}
$$

and

$$
\begin{aligned}
& \phi_{0}\left(c_{i}^{2} V\right) b_{s-j+1}(V)-c_{i} \phi_{1}\left(c_{i}^{2} V\right) \bar{b}_{s-j+1}(V)+a_{i j}(V) \\
& =\int_{0}^{1}\left(1-c_{i}-z\right) \phi_{1}\left(\left(1-c_{i}-z\right)^{2} V\right) l_{s-j+1}(z) \mathrm{d} z+\int_{0}^{c_{i}}\left(c_{i}-z\right) \phi_{1}\left(\left(c_{i}-z\right)^{2} V\right) l_{i}(z) \mathrm{d} z \\
& =\int_{0}^{1}\left(\tau-c_{i}\right) \phi_{1}\left(\left(\tau-c_{i}\right)^{2} V\right) l_{j}(\tau) \mathrm{d} \tau+\int_{0}^{c_{i}}\left(c_{i}-z\right) \phi_{1}\left(\left(c_{i}-z\right)^{2} V\right) l_{i}(z) \mathrm{d} z \\
& =\int_{1-c_{s-i+1}}^{1}\left(\tau-1+c_{s-i+1}\right) \phi_{1}\left(\left(\tau-1+c_{s-i+1}\right)^{2} V\right) l_{j}(\tau) \mathrm{d} \tau \\
& =\int_{0}^{c_{s-i+1}}\left(c_{s-i+1}-z\right) \phi_{1}\left(\left(c_{s-i+1}-z\right)^{2} V\right) l_{j}(1-z) \mathrm{d} z \\
& =\int_{0}^{c_{s-i+1}}\left(c_{s-i+1}-z\right) \phi_{1}\left(\left(c_{s-i+1}-z\right)^{2} V\right) l_{s-j+1}(z) \mathrm{d} z \\
& =a_{s-i+1, s-j+1}(V) .
\end{aligned}
$$

Therefore, (2.15) is identical to the following

$$
\begin{aligned}
U_{*}^{n i}= & \phi_{0}\left(\left(1-c_{i}\right)^{2} V\right) u^{n}+\left(1-c_{i}\right) h \phi_{1}\left(\left(1-c_{i}\right)^{2} V\right) \dot{u}^{n} \\
& +h^{2} \sum_{j=1}^{s} a_{s-i+1, s-j+1}(V) f\left(t_{n}+c_{s-j+1} h, U_{*}^{n i}\right) .
\end{aligned}
$$


Replacing all indicates $i$ and $j$ in (2.13), (2.14) and (2.16) by $s-i+1$ and $s-j+1$, respectively, we find that the ERKN method (2.4) with weights (2.5) and (2.6) is identical to its adjoint method consisted of (2.13), (2.14) and (2.16). Therefore, the ERKN methods determined by (2.4) are symmetric. This completes the proof of the theorem.

\section{Main theoretical results}

In this section, we state the error bounds of our continuous trigonometric collocation polynomial approximations for solving the hyperbolic system (1.1). The single step error bounds and the long-term convergence of continuous trigonometric collocation polynomial approximations (2.1) will be rigorously analysed. Our analysis will be based on the variation-of-constants formula (1.2) or (1.4) provided for the hyperbolic system (1.1). Before presenting our theoretical analysis, we need the following hypotheses on the regularity of $u$ and the nonlinearity of $f$.

Assumption 3.1 Suppose that the exact solution $u$ of the hyperbolic system (1.1) satisfies $u \in C^{s}\left(\left[t_{0}, T\right] ; \mathcal{X}\right)$ and the nonlinear function $f:\left[t_{0}, T\right] \times \mathcal{X} \rightarrow \mathcal{X}$ is Fréchet differentiable along a strip of the exact solution $u$. We also assume that all occurring derivatives are uniformly bounded.

Assumption 3.2 Let $f(t, u)$ be Lipschitz-continuous with respect to $u$, i.e., there exists a real number $L$ such that

$$
\|f(t, v(t))-f(t, w(t))\| \leq L\|v(t)-w(t)\|
$$

for all $t \in\left[t_{0}, T\right]$ and $\max (\|v(t)-u(t)\|,\|w(t)-u(t)\|) \leq$ Const.

Remark 3.1 Under Assumptions 3.1, it should be noted that the composition:

$$
g:\left[t_{0}, T\right] \rightarrow \mathcal{X}, \quad \text { i.e., } \quad t \rightarrow g(t)=f(t, u(t))
$$

is also Fréchet differentiable in a strip along the exact solution $u(t)$. Therefore, when $u \in C^{l}\left(\left[t_{0}, T\right], \mathcal{X}\right)$ and $g^{(l)} \in L^{\infty}\left(\left[t_{0}, T\right] ; \mathcal{X}\right)$ for $l=s, s+1, \ldots, 2 s$, the following assumptions are valid

$$
\sum_{l=0}^{m} \max _{t_{0} \leq t \leq T}\left\|g^{(s+l)}(t)\right\| \leq K_{m}, \quad m=0,1,2, \ldots, s,
$$

where $K_{m}, m=0,1,2, \ldots, s$ are constants and independent of $h$ and $A$.

Remark 3.2 Here, we remark that the essence of the continuous collocation polynomial approximation is to find the polynomial $y(t)$ in the Banach space $(\mathcal{X},\|\cdot\|)$. The 
polynomial $y(t)$ is always bounded over the interval $\left[t_{0}, T\right]$, and this implies that there exists a constant $M>0$ such that

$$
\max _{t_{0} \leq t \leq T}\|y(t)\| \leq M .
$$

Therefore, Assumption 3.2 is reasonable.

\subsection{Error estimates}

Theorem 3.1 (Single step error bounds I) Suppose that Assumptions 3.1 - 3.2 are satisfied and $g^{(l)} \in L^{\infty}\left(\left[t_{0}, T\right] ; \mathcal{X}\right)$ for $l=s, s+1, \ldots, 2 s$. Then, when the time step $h$ satisfies the limitation $0<h \leq \sqrt{\frac{1}{2 s L}}$, we have the error estimations for a single step:

(i) Under the local assumptions of $y\left(t_{n}\right)=u\left(t_{n}\right), \dot{y}\left(t_{n}\right)=\dot{u}\left(t_{n}\right)$, the single step error bounds of the continuous trigonometric collocation polynomial $y(t)$ defined by Definition 2.1 satisfy:

$$
\begin{gathered}
\|u(t)-y(t)\| \leq C_{1} h^{s+2} \quad \text { and } \\
\|\dot{u}(t)-\dot{y}(t)\| \leq C_{1} h^{s+1}, \quad \forall t \in\left[t_{n}, t_{n}+h\right] .
\end{gathered}
$$

(ii) Furthermore, if the collocation polynomial $y(t)$ satisfies $y^{(m)}\left(t_{n}\right)=u^{(m)}\left(t_{n}\right)$ for $m=1, \ldots, s$, then the derivatives of $y(t)$ satisfy the following estimations:

$$
\begin{aligned}
& \left\|u^{(m)}(t)-y^{(m)}(t)\right\| \leq C_{2} h^{s+2-m} \quad \text { and } \\
& \left\|u^{(m+1)}(t)-y^{(m+1)}(t)\right\| \leq C_{2} h^{s+1-m}, \quad \forall t \in\left[t_{n}, t_{n}+h\right] .
\end{aligned}
$$

Here, we point out that the constants $C_{1}$ and $C_{2}$ satisfy

$$
2 K_{0} \leq C_{1} \quad \text { and } \max _{1 \leq m \leq s}\left(2 K_{0} L \sum_{i=1}^{s} \max _{0 \leq z \leq 1}\left|l_{i}^{(m)}(z)\right|+K_{m}\right) \leq C_{2}
$$

and are obviously independent of $h$ and $A$.

Proof According to the definition of the continuous trigonometric collocation polynomial defined by Definition 2.1, it is clear that the polynomial $y(t)$ satisfies

$$
\ddot{y}\left(t_{n}+z h\right)=-A y\left(t_{n}+z h\right)+\sum_{i=1}^{s} l_{i}(z) f\left(t_{n}+c_{i} h, y\left(t_{n}+c_{i} h\right)\right) .
$$

Moreover, it is evident that the exact solution of (1.1) satisfies 


$$
\ddot{u}\left(t_{n}+z h\right)=-A u\left(t_{n}+z h\right)+\sum_{i=1}^{s} l_{i}(z) f\left(t_{n}+c_{i} h, u\left(t_{n}+c_{i} h\right)\right)+h^{s} E_{n}(z, h),
$$

where the interpolation error $E_{n}(z, h)=\frac{1}{s !} w_{s}(z) g_{s}^{(s)}\left(t_{n}+\xi^{n} h\right)$ for some $\xi^{n} \in\left(\min \left\{z, c_{1}, \ldots, c_{s}\right\}, \max \left\{z, c_{1}, \ldots, c_{s}\right\}\right)$ and $w_{s}(z)=\prod_{i=1}\left(z-c_{i}\right)$. In the light of Assumption $3.1, E_{n}(z, h)$ and its derivatives satisfy the estimations

$$
\left\|E_{n}^{(m)}(z, h)\right\| \leq \sum_{l=0}^{m} \max _{t_{0} \leq t \leq T}\left\|g^{(s+l)}(t)\right\| \leq K_{m}, \quad m=0,1,2, \ldots, s .
$$

Then, subtracting (3.4) from (3.5) leads to

$$
\ddot{e}\left(t_{n}+z h\right)=-A e\left(t_{n}+z h\right)+\sum_{i=1}^{s} l_{i}(z) \Delta f^{n i}+h^{s} E_{n}(z, h),
$$

where $\quad e\left(t_{n}+z h\right)=u\left(t_{n}+z h\right)-y\left(t_{n}+z h\right)$ and $\Delta f^{n i}=f\left(t_{n}+c_{i} h, u\left(t_{n}+c_{i} h\right)\right)-f\left(t_{n}+c_{i} h, y\left(t_{n}+c_{i} h\right)\right)$.

(i) Applying the variation-of-constants formula to the Eq. (3.6), we obtain

$$
\left\{\begin{array}{c}
e\left(t_{n}+z h\right)=\phi_{0}\left(z^{2} V\right) e\left(t_{n}\right)+z h \phi_{1}\left(z^{2} V\right) \dot{e}\left(t_{n}\right) \\
\quad+h^{2} \sum_{i=1}^{s} \int_{0}^{z}(z-\tau) \phi_{1}\left((z-\tau)^{2} V\right) l_{i}(\tau) \mathrm{d} \tau \Delta f^{n i} \\
\quad+h^{s+2} \int_{0}^{z}(z-\tau) \phi_{1}\left((z-\tau)^{2} V\right) E_{n}(\tau, h) \mathrm{d} \tau, \\
\dot{e}\left(t_{n}+z h\right)=-z h A \phi_{1}\left(z^{2} V\right) e\left(t_{n}\right)+\phi_{0}\left(z^{2} V\right) \dot{e}\left(t_{n}\right) \\
\quad+h \sum_{i=1}^{s} \int_{0}^{z} \phi_{0}\left((z-\tau)^{2} V\right) l_{i}(\tau) \mathrm{d} \tau \Delta f^{n i} \\
+h^{s+1} \int_{0}^{z} \phi_{0}\left((z-\tau)^{2} V\right) E_{n}(\tau, h) \mathrm{d} \tau .
\end{array}\right.
$$

Taking norms on both sides of Eqs. (3.7) and using Assumption 3.2 and the local assumptions $y\left(t_{n}\right)=u\left(t_{n}\right), \dot{y}\left(t_{n}\right)=\dot{u}\left(t_{n}\right)$, we obtain

$$
\left\|e\left(t_{n}+z h\right)\right\| \leq h^{2} s L \max _{0 \leq z \leq 1}\left\|e\left(t_{n}+z h\right)\right\|+K_{0} h^{s+2}
$$

and

$$
\left\|\dot{e}\left(t_{n}+z h\right)\right\| \leq h s L \max _{0 \leq z \leq 1}\left\|e\left(t_{n}+z h\right)\right\|+K_{0} h^{s+1} .
$$

If the time stepsize $h$ satisfies $h^{2} s L \leq \frac{1}{2}$, namely, $h \leq \sqrt{\frac{1}{2 s L}}$, then we have

$$
\max _{0 \leq z \leq 1}\left\|e\left(t_{n}+z h\right)\right\| \leq 2 K_{0} h^{s+2}
$$

Inserting the result (3.10) into the inequality (3.9) leads to 
$\left\|\dot{e}\left(t_{n}+z h\right)\right\| \leq h^{s+1}\left(2 K_{0} h^{2} s L+K_{0}\right) \leq 2 K_{0} h^{s+1}$.

Thus, let $C_{1} \geq 2 K_{0}$ in the above results, and then the proof of the first statement is complete.

(ii) The proof of the second statement follows from applying the variation-ofconstants formula to $h^{m} e^{(m+2)}\left(t_{n}+z h\right)=-A h^{m} e^{(m)}\left(t_{n}+z h\right)+\sum_{i=1}^{s} l_{i}^{(m)}(z) \Delta f^{n i}+h^{s} E^{(m)}(z, h)$, i.e.,

$$
\left\{\begin{array}{l}
h^{m} e^{(m)}\left(t_{n}+z h\right)=h^{m} \phi_{0}\left(z^{2} V\right) e^{(m)}\left(t_{n}\right) \\
\quad+z h^{m+1} \phi_{1}\left(z^{2} V\right) e^{(m+1)}\left(t_{n}\right)+h^{2} \sum_{i=1}^{s} \int_{0}^{z}(z-\tau) \phi_{1}\left((z-\tau)^{2} V\right) l_{i}^{(m)}(\tau) \mathrm{d} \tau \Delta f^{n i} \\
\quad+h^{s+2} \int_{0}^{z}(z-\tau) \phi_{1}\left((z-\tau)^{2} V\right) E^{(m)}(\tau, h) \mathrm{d} \tau, \\
h^{m} e^{(m+1)}\left(t_{n}+z h\right)=-z h^{m+1} A \phi_{1}\left(z^{2} V\right) e^{(m)}\left(t_{n}\right) \\
\quad+h^{m} \phi_{0}\left(z^{2} V\right) e^{(m+1)}\left(t_{n}\right)+h \sum_{i=1}^{s} \int_{0}^{z} \phi_{0}\left((z-\tau)^{2} V\right) l_{i}^{(m)}(\tau) \mathrm{d} \tau \Delta f^{n i} \\
\quad+h^{s+1} \int_{0}^{z} \phi_{0}\left((z-\tau)^{2} V\right) E^{(m)}(\tau, h) \mathrm{d} \tau .
\end{array}\right.
$$

Thus, the local assumptions $y^{(m)}\left(t_{n}\right)=u^{(m)}\left(t_{n}\right)$ for $m=1,2, \ldots, s$ lead to

$$
\begin{aligned}
& h^{m}\left\|e^{(m)}\left(t_{n}+z h\right)\right\| \leq h^{2} L \sum_{i=1}^{s} \max _{0 \leq z \leq 1}\left|l_{i}^{(m)}(z)\right| \\
& \quad \times \max _{0 \leq z \leq 1}\left\|e\left(t_{n}+z h\right)\right\|+K_{m} h^{s+2}
\end{aligned}
$$

and

$$
\begin{aligned}
& h^{m}\left\|e^{(m+1)}\left(t_{n}+z h\right)\right\| \leq h L \sum_{i=1}^{s} \max _{0 \leq z \leq 1}\left|l_{i}^{(m)}(z)\right| \\
& \times \max _{0 \leq z \leq 1}\left\|e\left(t_{n}+z h\right)\right\|+K_{m} h^{s+1} .
\end{aligned}
$$

Inserting the results (3.2) into the left sides of (3.11) and (3.12) and choosing the constant $C_{2}$ to satisfy

$$
\max _{1 \leq m \leq s}\left(2 K_{0} h^{2} L \sum_{i=1}^{s} \max _{0 \leq z \leq 1}\left|l_{i}^{(m)}(z)\right|+K_{m}\right) \leq C_{2},
$$

we obtain

$$
\begin{gathered}
\left\|e^{(m)}\left(t_{n}+z h\right)\right\| \leq C_{2} h^{s+2-m} \text { and }\left\|\dot{e}^{(m+1)}\left(t_{n}+z h\right)\right\| \leq C_{2} h^{s+1-m}, \\
m=1,2, \ldots, s .
\end{gathered}
$$

This completes the proof. 
Actually, Theorem 3.1 demonstrate the local error bounds for the continuous collocation polynomial approximations (2.1). In what follows, we will investigate the longterm convergence of the continuous collocation polynomial approximations. For this purpose, we rewritten the Eq. (3.7) as the following compact form:

$$
\begin{aligned}
{\left[\begin{array}{c}
\Omega e\left(t_{n}+z h\right) \\
\dot{e}\left(t_{n}+z h\right)
\end{array}\right]=} & \Psi(z, 0, V)\left[\begin{array}{c}
\Omega e\left(t_{n}\right) \\
\dot{e}\left(t_{n}\right)
\end{array}\right]+h \sum_{i=1}^{s} \int_{0}^{z} \Psi(z, \tau, V) l_{i}(\tau) \mathrm{d} \tau\left[\begin{array}{c}
0 \\
\Delta f^{n i}
\end{array}\right] \\
& +h^{s+1} \int_{0}^{z} \Psi(z, \tau, V)\left[\begin{array}{c}
0 \\
E_{n}(\tau, h)
\end{array}\right] \mathrm{d} \tau,
\end{aligned}
$$

where $\Psi(z, \tau, V)$ is defined as:

$$
\Psi(z, \tau, V)=\left[\begin{array}{cc}
\phi_{0}\left((z-\tau)^{2} V\right) & h(z-\tau) \Omega \phi_{1}\left((z-\tau)^{2} V\right) \\
-h(z-\tau) \Omega \phi_{1}\left((z-\tau)^{2} V\right) & \phi_{0}\left((z-\tau)^{2} V\right)
\end{array}\right],
$$

that is, $\Psi(z, \tau, V)$ is the rotation operator

$$
\Psi(z, \tau, V)=\left[\begin{array}{cc}
\cos ((z-\tau) h \Omega) & \sin ((z-\tau) h \Omega) \\
-\sin ((z-\tau) h \Omega) & \cos ((z-\tau) h \Omega)
\end{array}\right] .
$$

It is easy to see that the norm of $\Psi(z, \tau, V)$ satisfies:

$$
\|\Psi(z, \tau, V)\|=1, \quad \forall z, \tau \in[0,1] .
$$

This will be repeatedly used in our following theoretical analysis. For simplicity of analysis, we introduce the energy norm $\|\mid(u(t), \dot{u}(t))\| \|$ for the solutions $(u(t), \dot{u}(t)) \in L^{\infty}\left(\left[t_{0}, T\right] ; \mathcal{X}\right)$, i.e.,

$$
\|(u(t), \dot{u}(t))\|\|=\| u(t) \|+\sqrt{\|\dot{u}(t)\|^{2}+\|\Omega u(t)\|^{2}} .
$$

Moreover, we quote the following Gronwall inequality, which will play an important role in the convergence analysis.

Lemma 3.1 (See, e.g. [22, 23]) Let $\lambda$ be positive, $a_{k}, b_{k}, k \in \mathbb{N}$, be nonnegative and assume further that

$$
a_{k} \leq(1+\lambda \Delta t) a_{k-1}+\Delta t b_{k}, \quad k \in \mathbb{N} .
$$

Then

$$
a_{k} \leq \exp (\lambda k \Delta t)\left(a_{0}+\Delta t \sum_{m=1}^{k} b_{m}\right), \quad k \in \mathbb{N} .
$$

Now, we will show that the continuous collocation polynomial approximation defined by Definition 2.1 with nodes $\left\{c_{i}\right\}_{i=1}^{s}$ is of order $s$ at least. 
Theorem 3.2 (Long-term convergence I) Suppose that the Assumptions 3.1-3.2 are satisfied and $g^{(s)} \in L^{\infty}\left(\left[t_{0}, T\right] ; \mathcal{X}\right)$. Then, under the limitation of the time stepsize $0<h \leq \sqrt{\frac{1}{2 s L}}$, we obtain the error estimations:

$$
\|y(t)-u(t)\| \leq C_{4} h^{s} \quad \text { and } \quad\|\dot{y}(t)-\dot{u}(t)\| \leq C_{4} h^{s}, \quad \forall t \in\left[t_{0}, T\right],
$$

where $y(t)$ is the collocation polynomial of degree $s+1$ and the constant $C_{4}$ independent of $n, h$ and $A$.

Proof For any $t \in\left[t_{0}, T\right]$, there exists a nonnegative integer $n \in \mathbb{N}$ such that $t=t_{n}+z h, \forall z \in[0,1]$. It follows from taking norms on both sides of Eq. (3.13) and the first equation in (3.7) that:

$$
\begin{aligned}
\sqrt{\left\|\dot{e}\left(t_{n}+z h\right)\right\|^{2}+\left\|\Omega e\left(t_{n}+z h\right)\right\|^{2}} & \leq \sqrt{\left\|\dot{e}\left(t_{n}\right)\right\|^{2}+\left\|\Omega e\left(t_{n}\right)\right\|^{2}} \\
& +h s L \max _{0 \leq z \leq 1}\left\|e\left(t_{n}+z h\right)\right\|+K_{0} h^{s+1},
\end{aligned}
$$

and

$$
\left\|e\left(t_{n}+z h\right)\right\| \leq\left\|e\left(t_{n}\right)\right\|+z h\left\|\dot{e}\left(t_{n}\right)\right\|+h^{2} s L \max _{0 \leq z \leq 1}\left\|e\left(t_{n}+z h\right)\right\|+K_{0} h^{s+2} .
$$

Let the time stepsize $h_{0}$ be sufficiently small and meet the condition $h_{0}^{2} s L \leq \frac{1}{2}$, i.e., $h_{0} \leq \sqrt{\frac{1}{2 s L}}$. Then we have

$$
\max _{0 \leq z \leq 1}\left\|e\left(t_{n}+z h\right)\right\| \leq 2\left(\left\|e\left(t_{n}\right)\right\|+z h\left\|\dot{e}\left(t_{n}\right)\right\|+K_{0} h^{s+2}\right) .
$$

Inserting the result (3.18) into the inequality obtained by adding (3.16) and (3.17) leads to

$$
\begin{gathered}
\|\|\left(e\left(t_{n}+z h\right), \dot{e}\left(t_{n}+z h\right)\right)\|\mid \leq(1+h(1+4 s L)) \cdot\|\left\|\left(e\left(t_{n}\right), \dot{e}\left(t_{n}\right)\right)\right\| \|+4 K_{0} h^{s+1}, \\
\forall z \in[0,1] .
\end{gathered}
$$

Letting $z=1$ in (3.19) and using the Gronwall's inequality (see Lemma 3.1) to it, we have

$$
\|\|\left(e\left(t_{n}+h\right), \dot{e}\left(t_{n}+h\right)\|\| \leq \exp ((1+4 s L) T)\left(\|\|\left(e\left(t_{0}\right), \dot{e}\left(t_{0}\right)\|\|+4 T K_{0} h^{s}\right), \quad n=0,1,2, \ldots\right.\right.
$$

Therefore, there exist a constant $C_{3} \geq 4 T K_{0} \exp ((1+4 s L) T)$ such that

$$
\left\|e\left(t_{n}\right)\right\| \leq C_{3} h^{s}, \quad\left\|\dot{e}\left(t_{n}\right)\right\| \leq C_{3} h^{s}, \quad\left\|\Omega e\left(t_{n}\right)\right\| \leq C_{3} h^{s} .
$$

Furthermore, inserting the results (3.20) into (3.19) yields

$$
\left\|e\left(t_{n}+z h\right)\right\| \leq C_{4} h^{s} \quad \text { and } \quad\left\|\dot{e}\left(t_{n}+z h\right)\right\| \leq C_{4} h^{s}, \quad \forall z \in[0,1] .
$$

Obviously, the constant $C_{4}$ is independent on $n, h$ and $A$. Therefore, the theorem is confirmed. 


\subsection{Superconvergence of continuous trigonometric collocation polynomial approximations}

In the light of the results shown in Theorem 3.1 and Theorem 3.2, the term $\max _{0 \leq z \leq 1}\left|w_{s}(z)\right|=\max _{0 \leq z \leq 1} \prod_{i=1}^{s}\left|z-c_{i}\right|$ appears in the error bounds. This observation implies that by choosing suitable collocation nodes $c_{i}$, such as the zeros of the shifted Legendre polynomial, can minimize the error bounds to achieve a higher convergence order. In our numerical experiments, we will choose the collocation nodes $c_{i}$ as the zeros of the shifted Legendre polynomials

$$
\frac{\mathrm{d}^{s}}{\mathrm{~d} x^{s}}\left(x^{s}(1-x)^{s}\right) \quad \text { and } \quad \frac{\mathrm{d}^{s-2}}{\mathrm{~d} x^{s-2}}\left(x^{s-1}(1-x)^{s-1}\right)
$$

to generate the Gauss-type and Lobatto-type time integrators, respectively. The numerical results in Sect. 4 will show that the continuous trigonometric collocation polynomial approximation can be of superconvergence. In this subsection, we will present the rigorous theoretical analysis of this superconvergence. To this end, we consider $y\left(t_{n}+z h\right)$ as the solution of the following perturbed differential equation:

$$
\ddot{y}\left(t_{n}+z h\right)=-A y\left(t_{n}+z h\right)+f\left(t_{n}+z h, y\left(t_{n}+z h\right)\right)+\delta\left(t_{n}+z h\right),
$$

where the remainder $\delta\left(t_{n}+z h\right)$ will vanish at the chosen collocation points, i.e., $\delta\left(t_{n}+c_{i} h\right)=0$ for $i=1,2, \ldots, s$. Subtracting the perturbed differential equation (3.21) from the Eq. (1.1) and applying the variation-of-constant formula to this result, we obtain after the linearisation that

$$
\left\{\begin{array}{l}
e\left(t_{n}+z h\right)=\phi_{0}\left(z^{2} V\right) e\left(t_{n}\right)+z h \phi_{1}\left(z^{2} V\right) \dot{e}\left(t_{n}\right) \\
\quad+h^{2} \int_{0}^{z}(z-\tau) \phi_{1}\left((z-\tau)^{2} V\right) \frac{\partial f}{\partial u}\left(t_{n}+\tau h, u\left(t_{n}+\tau h\right)\right) e\left(t_{n}+\tau h\right) \mathrm{d} \tau \\
\quad+h^{2} \int_{0}^{z}(z-\tau) \phi_{1}\left((z-\tau)^{2} V\right)\left(-\delta\left(t_{n}+\tau h\right)+r\left(t_{n}+\tau h\right)\right) \mathrm{d} \tau, \\
\dot{e}\left(t_{n}+z h\right)=-z h A \phi_{1}\left(z^{2} V\right) e\left(t_{n}\right)+\phi_{0}\left(z^{2} V\right) \dot{e}\left(t_{n}\right) \\
\quad+h \int_{0}^{z} \phi_{0}\left((z-\tau)^{2} V\right) \frac{\partial f}{\partial u}\left(t_{n}+\tau h, u\left(t_{n}+\tau h\right)\right) e\left(t_{n}+\tau h\right) \mathrm{d} \tau \\
\quad+h \int_{0}^{z} \phi_{0}\left((z-\tau)^{2} V\right)\left(-\delta\left(t_{n}+\tau h\right)+r\left(t_{n}+\tau h\right)\right) \mathrm{d} \tau,
\end{array}\right.
$$

where the remainder $r\left(t_{n}+z h\right)$ is of magnitude $\mathcal{O}\left(\left\|u\left(t_{n}+z h\right)-y\left(t_{n}+z h\right)\right\|^{2}\right)$. Since the defect $\delta\left(t_{n}+z h\right)$ vanishes at the collocation nodes $c_{1}, \ldots, c_{s}$, the integral related to $\left\|\delta\left(t_{n}+z h\right)\right\|$ is equal to its quadrature error, i.e.,

$$
\int_{0}^{1}\left\|\delta\left(t_{n}+\tau h\right)\right\| \mathrm{d} \tau=\mathcal{O}\left(h^{p}\right)
$$

where $p$ is the algebraic order of the quadrature formula corresponding to the nodes $c_{1}, \ldots, c_{s}$. In what follows, we will show that the precision of the trigonometric collocation integrators defined by Definition 2.1 could be improved by choosing suitable collocation nodes. 
Theorem 3.3 (Single step error bounds II) Suppose that the exact solution $u(t)$ of the system (1.1) satisfies the Assumption 3.1. Then, there exists a sufficiently small $h_{0}>0$, such that when the time step $h$ meets the limitation $0<h \leq h_{0}$, the single step error bounds of the collocation polynomial $y(t)$ further satisfy

$$
\begin{aligned}
& \left\|u\left(t_{n}+z h\right)-y\left(t_{n}+z h\right)\right\|=\mathcal{O}\left(h^{p+2}\right) \quad \text { and } \\
& \left\|\dot{u}\left(t_{n}+z h\right)-\dot{y}\left(t_{n}+z h\right)\right\|=\mathcal{O}\left(h^{p+1}\right),
\end{aligned}
$$

where $s \leq p \leq 2 s$ is the algebraic order of the quadrature formula corresponding to the nodes $c_{1}, \ldots, c_{s}$.

Proof It follows from taking norms on both sides of (3.22) that

$$
\begin{aligned}
\left\|e\left(t_{n}+z h\right)\right\| \leq & h^{2} \int_{0}^{z}|z-\tau| \cdot\left\|\frac{\partial f}{\partial u}\left(t_{n}+\tau h, u\left(t_{n}+\tau h\right)\right)\right\| \cdot\left\|e\left(t_{n}+\tau h\right)\right\| \mathrm{d} \tau \\
& +h^{2} \int_{0}^{z}|z-\tau| \cdot\left(\left\|\delta\left(t_{n}+\tau h\right)\right\|+\left\|r\left(t_{n}+\tau h\right)\right\|\right) \mathrm{d} \tau \\
\leq & K_{0} h^{2} \max _{0 \leq z \leq 1}\left\|e\left(t_{n}+z h\right)\right\|+h^{2} \int_{0}^{1} \\
& \times\left(\left\|\delta\left(t_{n}+\tau h\right)\right\|+\left\|r\left(t_{n}+\tau h\right)\right\|\right) \mathrm{d} \tau
\end{aligned}
$$

and

$$
\begin{aligned}
\left\|\dot{e}\left(t_{n}+z h\right)\right\| \leq & h \int_{0}^{z}\left\|\frac{\partial f}{\partial u}\left(t_{n}+\tau h, u\left(t_{n}+\tau h\right)\right)\right\| \cdot\left\|e\left(t_{n}+\tau h\right)\right\| \mathrm{d} \tau \\
& +h \int_{0}^{z}\left(\left\|\delta\left(t_{n}+\tau h\right)\right\|+\left\|r\left(t_{n}+\tau h\right)\right\|\right) \mathrm{d} \tau \\
\leq & K_{0} h \max _{0 \leq z \leq 1}\left\|e\left(t_{n}+z h\right)\right\|+h \int_{0}^{1} \\
& \times\left(\left\|\delta\left(t_{n}+\tau h\right)\right\|+\left\|r\left(t_{n}+\tau h\right)\right\|\right) \mathrm{d} \tau .
\end{aligned}
$$

Let the stepsize $h$ satisfy $h \leq \sqrt{\frac{1}{2 K_{0}}}$. The inequality (3.25) leads to

$$
\left\|e\left(t_{n}+z h\right)\right\| \leq 2 h^{2} \int_{0}^{1}\left(\left\|\delta\left(t_{n}+\tau h\right)\right\|+\left\|r\left(t_{n}+\tau h\right)\right\|\right) \mathrm{d} \tau, \quad \forall z \in[0,1] .
$$

Inserting (3.27) into (3.26) yields

$$
\left\|\dot{e}\left(t_{n}+z h\right)\right\| \leq 2 h \int_{0}^{1}\left(\left\|\delta\left(t_{n}+\tau h\right)\right\|+\left\|r\left(t_{n}+\tau h\right)\right\|\right) \mathrm{d} \tau, \quad \forall z \in[0,1] .
$$


Moreover, the statements of Theorem 3.2 yield that the remainder $r\left(t_{n}+z h\right)$ is of the magnitude $\mathcal{O}\left(u\left(t_{n}+z h\right)-\left\|y\left(t_{n}+z h\right)\right\|^{2}\right)=\mathcal{O}\left(h^{2 s}\right)$. Therefore, if the order of the quadrature formula satisfies $s \leq p \leq 2 s$, we have

$$
\left\|u\left(t_{n}+z h\right)-y\left(t_{n}+z h\right)\right\|=\mathcal{O}\left(h^{p+2}\right) \quad \text { and } \quad\left\|\dot{u}\left(t_{n}+z h\right)-\dot{y}\left(t_{n}+z h\right)\right\|=\mathcal{O}\left(h^{p+1}\right) .
$$

By taking $h_{0} \leq \min \left\{\sqrt{\frac{1}{2 K_{0}}}, \sqrt{\frac{1}{2 s L}}\right\}$, the conclusion of the theorem is proved.

Theorem 3.4 (Long-term convergence II) Suppose that the collocation polynomial $y(t)$ of degree $s+1$ satisfies (2.1) and the exact solution $u(t)$ of the system (1.1) satisfies Assumption 3.1. Then, under the limitation of the time stepsize $h \leq \min \left\{\sqrt{\frac{1}{2 K_{0}}}, \sqrt{\frac{1}{2 s L}}\right\}$, we obtain the following superconvergence results

$$
\|u(t)-y(t)\|=\mathcal{O}\left(h^{p}\right) \quad \text { and } \quad\|\dot{u}(t)-\dot{y}(t)\|=\mathcal{O}\left(h^{p}\right), \quad \forall t \in\left[t_{0}, T\right],
$$

where $s \leq p \leq 2 s$ is the algebraic order of the quadrature formula corresponding to the nodes $c_{1}, \ldots, c_{s}$.

Proof It follows from rewriting the Eq. (3.22) as the following compact form that

$$
\begin{aligned}
& {\left[\begin{array}{c}
\Omega e\left(t_{n}+z h\right) \\
\dot{e}\left(t_{n}+z h\right)
\end{array}\right]=\Psi(z, 0, V)\left[\begin{array}{c}
\Omega e\left(t_{n}\right) \\
\dot{e}\left(t_{n}\right)
\end{array}\right]+h \int_{0}^{z} \Psi(z, \tau, V)} \\
& \times\left[\begin{array}{c}
0 \\
\frac{\partial f}{\partial u}\left(t_{n}+\tau h, u\left(t_{n}+\tau h\right)\right) e\left(t_{n}+\tau h\right)
\end{array}\right] \mathrm{d} \tau \\
& +h \int_{0}^{z} \Psi(z, \tau, V) \\
& \times\left[\begin{array}{c}
0 \\
-\delta\left(t_{n}+\tau h\right)+r\left(t_{n}+\tau h\right)
\end{array}\right] \mathrm{d} \tau .
\end{aligned}
$$

Taking norms on both sides of Eq. (3.30) gives

$$
\begin{gathered}
\sqrt{\left\|\dot{e}\left(t_{n}+z h\right)\right\|^{2}+\left\|\Omega e\left(t_{n}+z h\right)\right\|^{2}} \leq \sqrt{\left\|\dot{e}\left(t_{n}\right)\right\|^{2}+\left\|\Omega e\left(t_{n}\right)\right\|^{2}}+h K_{0} \max _{0 \leq z \leq 1}\left\|e\left(t_{n}+z h\right)\right\| \\
+h \int_{0}^{1}\left(\left\|\delta\left(t_{n}+z h\right)\right\|+\left\|r\left(t_{n}+z h\right)\right\|\right) \mathrm{d} z .
\end{gathered}
$$

Similarly, by taking norms on both sides of the first equation in (3.30), we have

$$
\begin{aligned}
\left\|e\left(t_{n}+z h\right)\right\| \leq & \left\|e\left(t_{n}\right)\right\|+z h\left\|\dot{e}\left(t_{n}\right)\right\|+h^{2} K_{0} \max _{0 \leq z \leq 1}\left\|e\left(t_{n}+z h\right)\right\| \\
& +h^{2} \int_{0}^{1}\left(\left\|\delta\left(t_{n}+z h\right)\right\|+\left\|r\left(t_{n}+z h\right)\right\|\right) \mathrm{d} z .
\end{aligned}
$$

It then follows from choosing the stepsize $h$ satisfies $h \leq \sqrt{\frac{1}{2 K_{0}}}$ that 


$$
\begin{aligned}
\max _{0 \leq z \leq 1}\left\|e\left(t_{n}+z h\right)\right\| \leq & 2\left(\left\|e\left(t_{n}\right)\right\|+h\left\|\dot{e}\left(t_{n}\right)\right\|\right) \\
& +2 h^{2} \int_{0}^{1}\left(\left\|\delta\left(t_{n}+z h\right)\right\|+\left\|r\left(t_{n}+z h\right)\right\|\right) \mathrm{d} z .
\end{aligned}
$$

Inserting (3.33) into the results obtained by adding up (3.31) and (3.32) yields that

$$
\begin{aligned}
\|\|\left(e\left(t_{n}+z h\right),\right. & \left.\dot{e}\left(t_{n}+z h\right)\right)\|\| \leq\|\|\left(e\left(t_{n}\right), \dot{e}\left(t_{n}\right)\right)\|\|+z h\left\|\dot{e}\left(t_{n}\right)\right\| \\
& +h(1+h) K_{0} \max _{0 \leq z \leq 1}\left\|e\left(t_{n}+z h\right)\right\| \\
& +h(1+h) \int_{0}^{1}\left(\left\|\delta\left(t_{n}+z h\right)\right\|+\left\|r\left(t_{n}+z h\right)\right\|\right) \mathrm{d} z \\
\leq & \left(1+h\left(1+4 K_{0}\right)\right) \cdot\|\|\left(e\left(t_{n}\right), \dot{e}\left(t_{n}\right)\right)\|\| \\
& +4 h \int_{0}^{1}\left(\left\|\delta\left(t_{n}+z h\right)\right\|+\left\|r\left(t_{n}+z h\right)\right\|\right) \mathrm{d} z .
\end{aligned}
$$

Taking $z=1$ in (3.34) and using the Gronwall's inequality (see Lemma 3.1) to the obtained result leads to

$$
\left\|\mid\left(e\left(t_{n}+h\right), \dot{e}\left(t_{n}+h\right)\right)\right\| \| \leq 4 h \exp \left(\left(1+4 K_{0}\right) T\right) \sum_{k=0}^{n} \int_{0}^{1}\left(\left\|\delta\left(t_{k}+z h\right)\right\|+\left\|r\left(t_{k}+z h\right)\right\|\right) \mathrm{d} z .
$$

$\begin{array}{lllll}\text { According to } & \text { Theorem } & 3.2 & \text { we }\end{array}$ $\left\|r\left(t_{n}+z h\right)\right\|=\mathcal{O}\left(\left\|u\left(t_{n}+z h\right)-y\left(t_{n}+z h\right)\right\|^{2}\right)=\mathcal{O}\left(h^{2 s}\right)$. Therefore, if the order of the quadrature formula satisfies $s \leq p \leq 2 s$, we obtain

$$
\left\|u\left(t_{n}\right)-y\left(t_{n}\right)\right\|=\mathcal{O}\left(h^{p}\right) \quad \text { and } \quad\left\|\dot{u}\left(t_{n}\right)-\dot{y}\left(t_{n}\right)\right\|=\mathcal{O}\left(h^{p}\right) .
$$

Inserting (3.35) into (3.34) yields

$$
\begin{aligned}
& \left\|u\left(t_{n}+z h\right)-y\left(t_{n}+z h\right)\right\|=\mathcal{O}\left(h^{p}\right) \quad \text { and } \\
& \left\|\dot{u}\left(t_{n}+z h\right)-\dot{y}\left(t_{n}+z h\right)\right\|=\mathcal{O}\left(h^{p}\right), \quad \forall z \in[0,1] .
\end{aligned}
$$

The statement of the theorem is confirmed.

Remark 3.3 If the nodes $\left\{c_{i}\right\}_{i=1}^{s}$ are chosen, from the analysis of Theorem 3.3 and Theorem 3.4, we have

$$
\int_{0}^{1}\left\|\delta\left(t_{n}+\tau h\right)\right\| \mathrm{d} \tau=\mathcal{O}\left(h^{p}\right) .
$$

This implies that the continuous trigonometric collocation polynomial approximations have the same order as the underlying quadrature formula. For instance, we take $\left\{c_{i}\right\}_{i=1}^{s}$ as zeros of the shifted Legendre polynomials

$$
\frac{\mathrm{d}^{s}}{\mathrm{~d} x^{s}}\left(x^{s}(1-x)^{s}\right) \quad \text { or } \quad \frac{\mathrm{d}^{s-2}}{\mathrm{~d} x^{s-2}}\left(x^{s-1}(1-x)^{s-1}\right)
$$


the accuracy of the continuous trigonometric collocation polynomial approximations can achieve order $2 s$ or $2 s-2$, respectively. The fact will be demonstrated again in our numerical experiments in Sect. 4.

\subsection{Long-term behaviour of energy conservation}

In this section, we turn to another essential property of the conservative hyperbolic system (1.1). It is easy to see that the hyperbolic system (1.1) has the following important conservative quantity:

$$
\begin{aligned}
H(u(t), \dot{u}(t)):= & \frac{1}{2}\|\dot{u}(t)\|^{2}+\frac{1}{2}\|\Omega u(t)\|^{2} \\
& +(F(t, u(t)), 1) \equiv H\left(u\left(t_{0}\right), \dot{u}\left(t_{0}\right)\right),
\end{aligned}
$$

where the inner product $(\cdot, \cdot)$ is corresponding to $\|\cdot\|$, and $F$ is the primitive function of $f$ defined as:

$$
F(t, s)=-\int_{0}^{s} f(t, \rho) \mathrm{d} \rho .
$$

In what follows, we will focus on analysing the long-term energy conservation of the continuous trigonometric collocation polynomial approximations for solving the hyperbolic system (1.1).

Theorem 3.5 Let $0 \leq c_{1}<c_{2}<\cdots<c_{s} \leq 1$ be the collocation nodes and $y(t)$ defined by Definition 2.1 be the collocation polynomial corresponding to the nodes. Then the energy defined in (3.38) satisfies the estimation

$$
\left|H(y(t), \dot{y}(t))-H\left(y\left(t_{0}\right), \dot{y}\left(t_{0}\right)\right)\right|=\mathcal{O}\left(h^{p}\right),
$$

where $t \in\left[t_{0}, T\right]$ and $s \leq p \leq 2 s$ is the algebraic order of the quadrature formula corresponding to the nodes.

Proof For any $t_{0} \leq t \leq T$, there exists a number $n \in \mathbb{N}$ such that $t=t_{n}+\mu h, \forall \mu \in[0,1]$. Therefore, the energy error can be expressed as

$$
\begin{aligned}
\mid H(y(t), \dot{y}(t)) & -H\left(y\left(t_{0}\right), \dot{y}\left(t_{0}\right)\right)|\leq| H\left(y\left(t_{n}+\mu h\right), \dot{y}\left(t_{n}+\mu h\right)\right)-H\left(y\left(t_{n}\right), \dot{y}\left(t_{n}\right)\right) \mid \\
& +\sum_{k=1}^{n}\left|H\left(y\left(t_{k}\right), \dot{y}\left(t_{k}\right)\right)-H\left(y\left(t_{k-1}\right), \dot{y}\left(t_{k-1}\right)\right)\right| .
\end{aligned}
$$

In order to obtain the estimation for the energy (3.38) over a long time interval $\left[t_{0}, T\right]$, we need to estimate $\left|H\left(y\left(t_{k}+\mu h\right), \dot{y}\left(t_{k}+\mu h\right)\right)-H\left(y\left(t_{k}\right), \dot{y}\left(t_{k}\right)\right)\right|$ for $k=0,1, \ldots, n$. Clearly, the collocation polynomial $y\left(t_{k}+\mu h\right)$ satisfies 


$$
\ddot{y}\left(t_{k}+\mu h\right)=-A y\left(t_{k}+\mu h\right)+f\left(t_{k}+\mu h, y\left(t_{k}+\mu h\right)\right)+\delta\left(t_{k}+\mu h\right) .
$$

For any $k=0,1, \ldots, n$, by taking the inner product of both sides of (3.41) with $y\left(t_{k}+\mu h\right)$, we have

$$
\begin{aligned}
\frac{\mathrm{d}}{\mathrm{d} t}\left(\frac{1}{2}\left\|\dot{y}\left(t_{k}+\mu h\right)\right\|^{2}+\frac{1}{2}\left\|\Omega y\left(t_{k}+\mu h\right)\right\|^{2}\right. \\
\left.\quad+\left(F\left(t_{k}+\mu h, y\left(t_{k}+\mu h\right)\right), 1\right)\right)=\left(\delta\left(t_{k}+\mu h\right), y\left(t_{k}+\mu h\right)\right) .
\end{aligned}
$$

Therefore, integrating the Eq. (3.42) for $\mu$ from 0 to $z$ and using the Hölder's inequality, we obtain

$$
\begin{aligned}
\mid H\left(y\left(t_{k}+z h\right), \dot{y}\left(t_{k}+z h\right)\right) & -H\left(y\left(t_{k}\right), \dot{y}\left(t_{k}\right)\right) \mid \leq h \int_{0}^{z}\left\|\delta\left(t_{k}+\mu h\right)\right\|_{*} \mathrm{~d} \mu \max _{t_{0} \leq t \leq T}\|y(t)\| \\
& \leq h M \int_{0}^{1}\left\|\delta\left(t_{k}+\mu h\right)\right\|_{*} \mathrm{~d} \mu .
\end{aligned}
$$

Here, $\|\cdot\|_{*}$ is the dual norm of $\|\cdot\|$ and $M$ is the bound of the collocation polynomial $y(t)$ over the interval $\left[t_{0}, T\right]$. Inserting the above results into (3.41) leads to

$$
\left|H(y(t), \dot{y}(t))-H\left(y\left(t_{0}\right), \dot{y}\left(t_{0}\right)\right)\right| \leq h M \sum_{k=0}^{n} \int_{0}^{1}\left\|\delta\left(t_{k}+\mu h\right)\right\|_{*} \mathrm{~d} \mu .
$$

Since the defect $\delta\left(t_{k}+\mu h\right)$ vanishes at the collocation nodes $c_{1}, \ldots, c_{s}$, the integral related to $\left\|\delta\left(t_{n}+\mu h\right)\right\|_{*}$ is also equal to its quadrature error, namely,

$$
\int_{0}^{1}\left\|\delta\left(t_{n}+\mu h\right)\right\|_{*} \mathrm{~d} \mu=\mathcal{O}\left(h^{p}\right)
$$

Therefore, we obtain the estimate of energy error as follows

$$
\left|H(y(t), \dot{y}(t))-H\left(y\left(t_{0}\right), \dot{y}\left(t_{0}\right)\right)\right|=\mathcal{O}\left(h^{p}\right) .
$$

The conclusion of the theorem is confirmed.

\section{Numerical experiments}

In this section, we will derive four practical continuous trigonometric collocation polynomial approximations and illustrate the numerical results for solving the KleinGordon equation, two-dimensional sine-Gordon equation, and Duffing equation. We report numerical results to support our error estimates and demonstrate the superiority of the continuous collocation polynomial approximation. The numerical results are really promising, which are powerful to verify our theoretical analysis results for our continuous trigonometric collocation polynomial approximations. 
- Trigonometric collocation polynomial approximation with Gauss nodes: By taking the nodes $c_{1}, \cdots, c_{s}$ as the zeros of the sth shifted Gauss-Legendre polynomial

$$
\frac{\mathrm{d}^{s}}{\mathrm{~d} x^{s}}\left(x^{s}(x-1)^{s}\right)
$$

then the Gauss quadrature formulas means that the integral (3.23) has order $p=2 s$. In this work, we choose the two-point Gauss-Legendre nodes

$$
c_{1}=\frac{3-\sqrt{3}}{6}, \quad c_{2}=\frac{3+\sqrt{3}}{6},
$$

and the three-point Gauss-Legendre nodes

$$
c_{1}=\frac{5-\sqrt{15}}{10}, \quad c_{2}=\frac{1}{2}, \quad c_{3}=\frac{5+\sqrt{15}}{10},
$$

to construct the fourth-order and sixth-order trigonometric collocation time integrators, which are denoted by GTC2s 4 and $G T C 3 s 6$, respectively.

- Trigonometric collocation polynomial approximation with Lobatto nodes: If we take the nodes $c_{1}, \cdots, c_{s}$ as the zeros of the $s$ th Legendre polynomial

$$
\frac{\mathrm{d}^{s-2}}{\mathrm{~d} x^{s-2}}\left(x^{s-1}(x-1)^{s-1}\right),
$$

then the Lobatto quadrature formulas lead the integral (3.23) could achieve the highest possible order $p=2 s-2$. Similarly, by taking the three-point Lobatto nodes

$$
c_{1}=0, \quad c_{2}=\frac{1}{2}, \quad c_{3}=1
$$

and the four-point Lobatto nodes

$$
c_{1}=0, \quad c_{2}=\frac{5-\sqrt{5}}{10}, \quad c_{3}=\frac{5+\sqrt{5}}{10}, \quad c_{4}=1,
$$

we can derive the fourth-order and sixth-order trigonometric collocation time integrators, and denoted as $L T C 2 s 4$ and $L T C 3 s 6$, respectively.

In order to demonstrate the superiority of the proposed integrators, we select the following time integrators for comparison:

- BH1: the symmetric Birkhoff-Hermite time integrator of order four derived in [22]

- BH2: the symmetric Birkhoff-Hermite time integrator of order six derived in [22]

- GAS2s4: the two-stage Gauss time integration method of order four presented in [16]; 
- GAS3s6: the three-stage Gauss time integration method of order six presented in [16];

- LIIIA3s4: the Labatto IIIA method of order four presented in [16];

- LIIIA4s6: the Labatto IIIA method of order six presented in [16].

- ERKN3s4: the three-stage explicit ERKN method of order four derived in [38];

- ERKN7s6: the seven-stage explicit ERKN method of order six presented in [33];

During the numerical experiments, it should be noted that the fixed-point iteration are used for all the implicit integrators. The iteration procedure will be stopped once the $l^{\infty}$ norm of the difference between two successive approximations is smaller than the fixed error tolerance $10^{-15}$. Here, we also point out that if the error produced by a method is too large for some time stepsize $h$, then the corresponding point will not be plotted in the figure. Moreover, we should point out that the ERKN3s4 and ERKN7s6 methods are quite different with our proposed methods in this paper. The chosen ERKN3s4 and ERKN7s6 methods are derived by solving the order conditions (see $[33,38]$ ). Our derived time integrators are yielded by using the continuous collocation polynomial approximation.

All computations in the numerical experiments are carried out by using MATLAB 2016b on the computer Lenovo ThinkCentre M8300t (CPU: Intel (R) Core (TM) i7-2400 CPU @ 3.10 GHz, Memory: 8 GB, Os: Microsoft Windows 10 with 64 bit).

Problem 4.1 Consider the dimensionless relativistic Klein-Gordon equation which is highly oscillatory in time (see, e.g. [2, 34, 37]):

$$
\left\{\begin{array}{l}
\varepsilon^{2} u_{t t}(x, t)-\Delta u(x, t)+\frac{1}{\varepsilon^{2}} u(x, t)+f(u(x, t))=0, \\
u(x, 0)=\psi_{1}(x), \quad u_{t}(x, 0)=\frac{1}{\varepsilon^{2}} \psi_{2}(x),
\end{array}\right.
$$

over the region $(x, t) \in[-L, L] \times[0, T]$ with the initial functions

$$
\psi_{1}(x)=\frac{2}{e^{x^{2}}+e^{-x^{2}}}, \quad \psi_{2}(x)=0
$$

and the cubic nonlinearity, i.e. $f(u)=4 u^{3}$. Here, the dimensionless parameter $\varepsilon$ is chosen as $\varepsilon=0.5$ and 0.1 , respectively. Moreover, we suppose that the Klein-Gordon equation (4.1) is equipped with the periodic boundary conditions. Therefore, the Fourier pseudo-spectral method will be used to discretise the spatial derivatives, and the Klein-Gordon equation (4.1) can be converted into the following form

$$
\varepsilon^{2} \frac{\mathrm{d}^{2}}{\mathrm{~d} t^{2}} U(t)+A U(t)+f(U(t))=0
$$

where $U(t)=\left(U_{1}(t), \ldots, U_{M}(t)\right)^{\top}$ with $U_{i}(t) \approx u\left(x_{i}, t\right)$, and $A=D_{2}+I / \varepsilon^{2}$ is an $M \times M$ matrix used to approximate the operator $-\Delta+1 / \varepsilon^{2}$. Here, $D_{2}$ is the secondorder symmetric semi-definite spectral differential matrix (see $[14,17,26,27]$ ), and $I$ is the unit matrix. The discrete energy is given by 


$$
H(U(t), \dot{U}(t))=\frac{\varepsilon^{2}}{2}\|\dot{U}(t)\|_{2}^{2}+\frac{1}{2}(A U(t), U(t))+\|U(t)\|_{4}^{4},
$$

where the discrete $l^{p}$ norms $\|\cdot\|_{p}$ for $p=2,4$ are defined as

$$
\|u\|_{p}^{p}=\Delta x \sum_{i=1}^{M}\left|u_{i}\right|^{p},
$$

the discrete inner product $(u, v)$ is defined by

$$
(u, v)=\Delta x \sum_{i=1}^{M} u_{i} \bar{v}_{i} .
$$

Here, $\Delta x=2 L / M$ is the spatial stepsize. In order to test the long-term numerical behaviour of the proposed time integrators, we set $L=30, T=100$ and fixed the spatial mesh size $M=1024$ for this problem. In addition, as is known, the exact solution of the Eq. (4.1) cannot be represented explicitly. Therefore, we use the posterior error, i.e., $R E=\|U(h ; T)-U(h / 2 ; T)\|_{2}$, to compute the convergence order.

In Tables 1 and 2, we test the numerical precision of the time integrators "GTC2s4", "GTC3s6", "LTC3s4" and "LTC4s6" with different $\varepsilon$. The numerical data indicate that the continuous trigonometric collocation polynomial approximations with Gauss points and Lobatto points can achieve 2 sth order and $(2 s-2)$ th order, respectively.

\begin{tabular}{|c|c|c|c|c|c|c|c|c|}
\hline \multirow[t]{2}{*}{$h$} & \multicolumn{2}{|l|}{ GTC2s4 } & \multicolumn{2}{|l|}{ GTC3s6 } & \multicolumn{2}{|l|}{ LTC $3 s 4$} & \multicolumn{2}{|l|}{ LTC4s6 } \\
\hline & Error & Rate & Error & Rate & Error & Rate & Error & Rate \\
\hline$h$ & $6.7910 \mathrm{E}-05$ & $*$ & $4.5151 \mathrm{E}-07$ & $*$ & $7.1473 \mathrm{E}-05$ & $*$ & $5.2011 \mathrm{E}-07$ & $*$ \\
\hline$h / 2$ & $4.0054 \mathrm{E}-06$ & 4.0836 & $5.9649 \mathrm{E}-09$ & 6.2421 & $4.1139 \mathrm{E}-06$ & 4.1188 & $7.1630 \mathrm{E}-09$ & 6.1821 \\
\hline$h / 2^{2}$ & $2.4725 \mathrm{E}-07$ & 4.0179 & $8.9788 \mathrm{E}-11$ & 6.0538 & $2.5238 \mathrm{E}-07$ & 4.0268 & $1.0944 \mathrm{E}-10$ & 6.0324 \\
\hline$h / 2^{3}$ & $1.5407 \mathrm{E}-08$ & 4.0043 & $1.4045 \mathrm{E}-12$ & 5.9984 & $1.5702 \mathrm{E}-08$ & 4.0066 & $1.7022 \mathrm{E}-12$ & 6.0065 \\
\hline
\end{tabular}

Table 1 Temporal precision of "GTC2s4", "GTC3s6", "LTC3s4" and "LTC4s6" for solving Problem 4.1 with $h=0.08$ and $\varepsilon=0.5$

*No data

\begin{tabular}{|c|c|c|c|c|c|c|c|c|}
\hline \multirow[t]{2}{*}{$h$} & \multicolumn{2}{|l|}{ GTC2s4 } & \multicolumn{2}{|l|}{ GTC3s6 } & \multicolumn{2}{|l|}{ LTC3s4 } & \multicolumn{2}{|l|}{ LTC4s6 } \\
\hline & Error & Rate & Error & Rate & Error & Rate & Error & Rate \\
\hline$h$ & $4.3034 \mathrm{E}-04$ & $*$ & $4.4094 \mathrm{E}-06$ & $*$ & $2.9664 \mathrm{E}-04$ & $*$ & $9.2841 \mathrm{E}-06$ & $*$ \\
\hline$h / 2$ & $2.3794 \mathrm{E}-05$ & 4.1768 & $1.0708 \mathrm{E}-08$ & 8.6857 & $2.3478 \mathrm{E}-05$ & 3.6593 & $9.1543 \mathrm{E}-08$ & 6.6642 \\
\hline$h / 2^{2}$ & $1.4706 \mathrm{E}-06$ & 4.0161 & $1.9491 \mathrm{E}-10$ & 5.7798 & $1.5089 \mathrm{E}-06$ & 3.9597 & $1.3729 \mathrm{E}-09$ & 6.0592 \\
\hline$h / 2^{3}$ & $9.1694 \mathrm{E}-08$ & 4.0034 & $3.2456 \mathrm{E}-12$ & 5.9082 & $9.4910 \mathrm{E}-08$ & 3.9908 & $2.1330 \mathrm{E}-11$ & 6.0081 \\
\hline
\end{tabular}

Table 2 Temporal precision of "GTC2s4", "GTC3s6", "LTC3s4" and "LTC4s6" for solving Problem 4.1 with $h=0.01$ and $\varepsilon=0.1$ 
The computational results in Tables 1 and 2 demonstrate that the temporal accuracy is completely consistent with our theoretical analysis. The proposed continuous trigonometric collocation polynomial approximations can be of superconvergence (see Theorem 3.4). The logarithms of the posterior errors, i.e., $\log _{10}(R E)$, are plotted in Figs. 1 and 2, in comparison with the classical time integrators "BH1", "BH2", "ERKN3s4", "ERKN7s6", "GAS2s4", "GAS3s6", "LIIIA3s4" and "LIIIA4s6". It can be observed from these two figures that the proposed continuous trigonometric collocation integrators are much more accurate than these traditional methods. The data in Tables 3 and 4 illustrate the precision of the discrete energy conservation, which is consistent with our theoretical analyse results in Theorem 3.5. The proposed temporal integrators have better long-term behaviour of energy conservation.

Problem 4.2 Consider the following sine-Gordon equations in two dimensions (see, e.g. $[22,23])$ :

$$
\left\{\begin{array}{l}
u_{t t}-\kappa^{2}\left(u_{x x}+u_{y y}\right)=-\sin (u), \quad(x, y) \in[-1,1] \times[-1,1], t \in[0,100], \\
u(x, y, 0)=4 \arctan \left(\exp \left(3-\sqrt{x^{2}+y^{2}} / \kappa^{2}\right)\right), \quad u_{t}(x, y, 0)=0
\end{array}\right.
$$
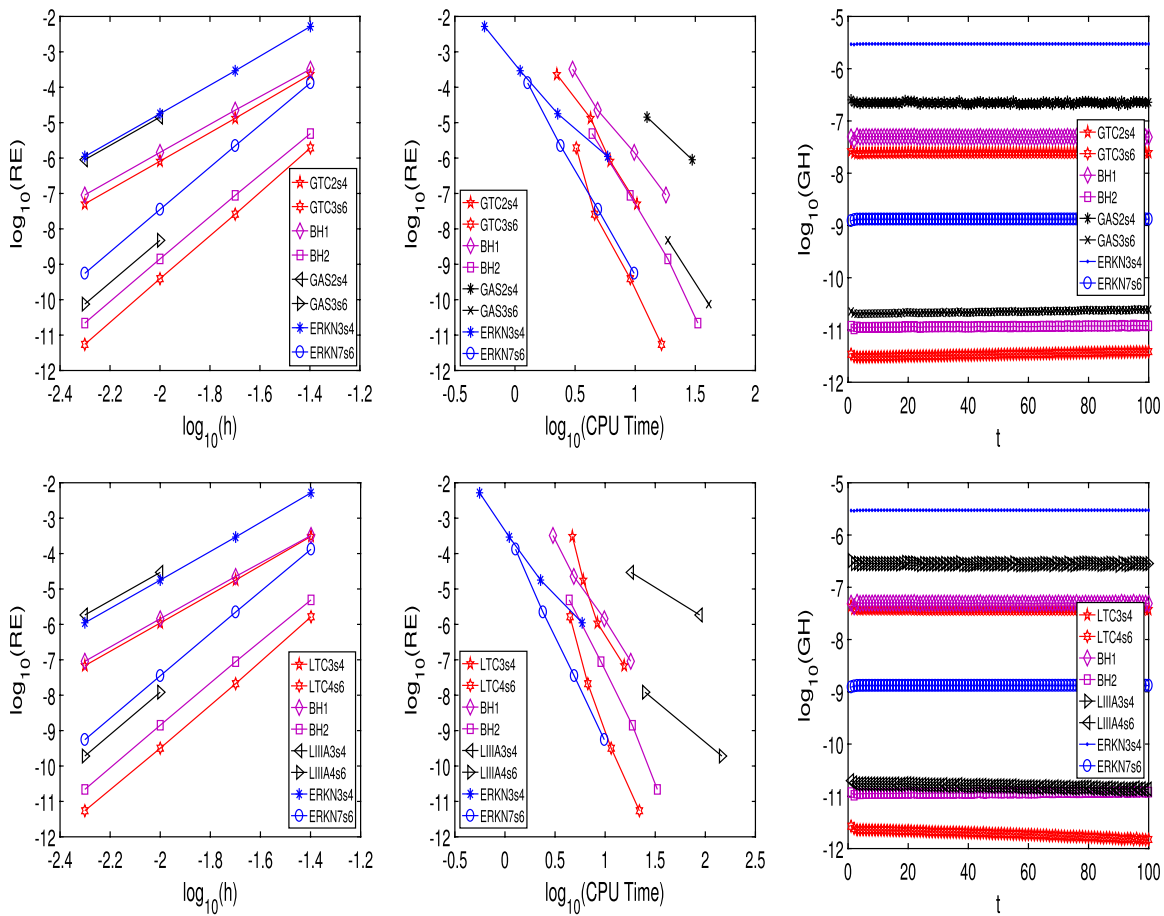

Fig. 1 Results for Problem 4.1 with $\varepsilon=0.5$. (Left) the $\log -\log$ plot of posterior error $R E$ against different time stepsizes $h$. (Middle) the $\log -\log$ plot of posterior error $R E$ against CPU time. (Right) the logarithm of the discrete energy error $G H$ against $t$ 

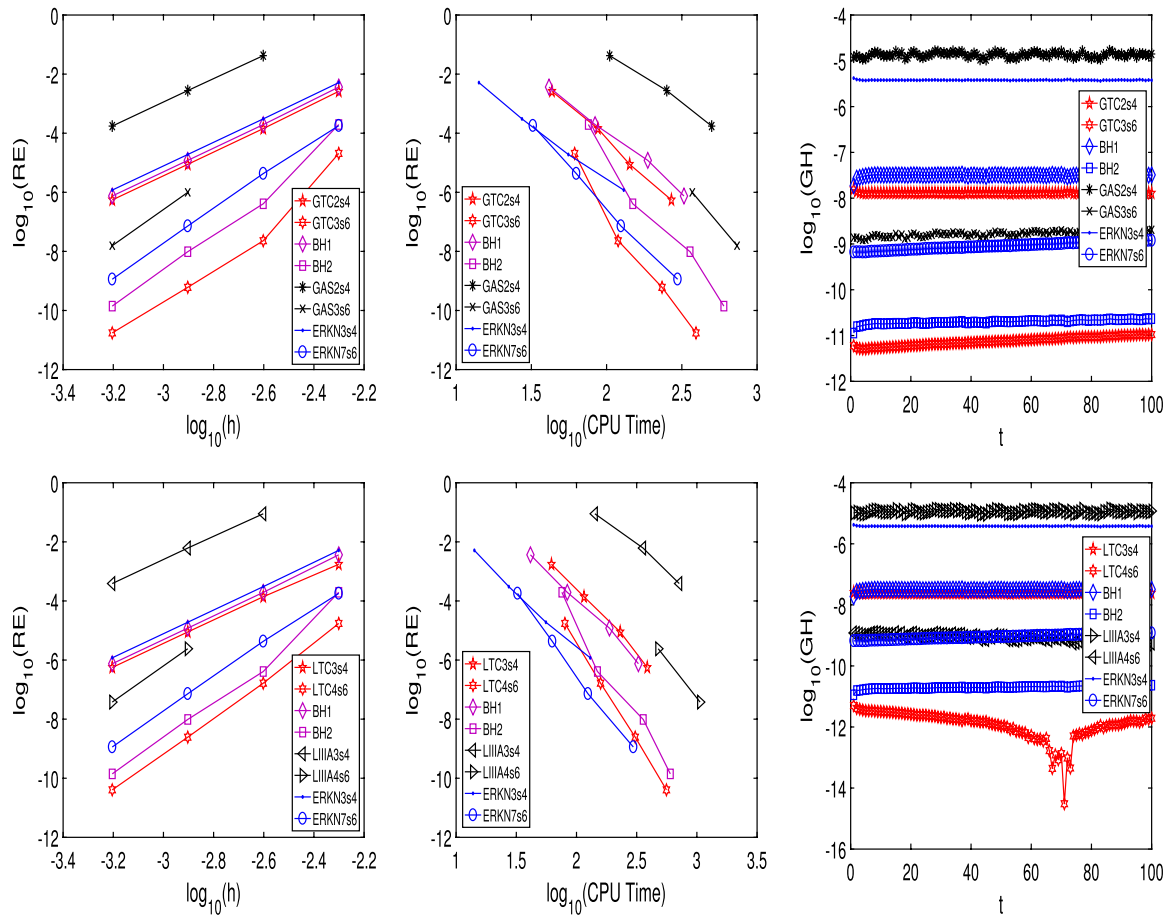

Fig. 2 Results for Problem 4.1 with $\varepsilon=0.1$. (Left) the $\log -\log$ plot of posterior error $R E$ against different time stepsizes $h$. (Middle) the $\log -\log$ plot of posterior error $R E$ against CPU time. (Right) the logarithm of the discrete energy error $G H$ against $t$

Table 3 Numerical precision of the preservation of the discrete energy for Problem 4.1 with $h=0.08$ and $\varepsilon=0.5$

\begin{tabular}{|c|c|c|c|c|c|c|c|c|}
\hline \multirow[t]{2}{*}{$h$} & \multicolumn{2}{|l|}{ GTC2s4 } & \multicolumn{2}{|l|}{ GTC3s6 } & \multicolumn{2}{|l|}{ LTC3s4 } & \multicolumn{2}{|l|}{ LTC4s6 } \\
\hline & Error & Rate & Error & Rate & Error & Rate & Error & Rate \\
\hline$h$ & $9.7402 \mathrm{E}-04$ & $*$ & $8.8434 \mathrm{E}-06$ & $*$ & $1.3353 \mathrm{E}-03$ & $*$ & $9.1889 \mathrm{E}-06$ & * \\
\hline$h / 2$ & $6.3235 E-05$ & 3.9452 & $1.3105 \mathrm{E}-07$ & 6.0764 & $8.1005 E-05$ & 4.0430 & $1.2996 \mathrm{E}-07$ & 6.1437 \\
\hline$h / 2^{2}$ & $3.8634 \mathrm{E}-06$ & 4.0328 & $1.9952 \mathrm{E}-09$ & 6.0375 & $5.0517 \mathrm{E}-06$ & 4.0032 & $2.0248 \mathrm{E}-09$ & 6.0042 \\
\hline$h / 2^{3}$ & $2.4042 \mathrm{E}-07$ & 4.0062 & $3.1321 \mathrm{E}-11$ & 5.9933 & $3.1464 \mathrm{E}-07$ & 4.0050 & $3.1393 \mathrm{E}-11$ & 6.0112 \\
\hline
\end{tabular}

*No data

where $\kappa^{2}$ is a dimensionless parameter. We also suppose that the two dimensional sine-Gordon equation (4.3) is subjected to periodic boundary conditions. The spatial derivatives are approximated by the two dimensional Fourier pseudo-spectral method. Therefore, the Eq. (4.3) can be converted to the following matrix form:

$$
\frac{\mathrm{d}^{2}}{\mathrm{~d} t^{2}} U(t)+D_{2}^{x} U(t)+U(t) D_{2}^{y}=-\sin (U(t))
$$


Table 4 Numerical precision of the preservation of the discrete energy for Problem 4.1 with $h=0.01$ and $\varepsilon=0.1$

\begin{tabular}{|c|c|c|c|c|c|c|c|c|}
\hline \multirow[t]{2}{*}{$h$} & \multicolumn{2}{|l|}{ GTC2s4 } & \multicolumn{2}{|l|}{ GTC3s6 } & \multicolumn{2}{|l|}{$\mathrm{LTC} 3 \mathrm{~s} 4$} & \multicolumn{2}{|l|}{ LTC4s6 } \\
\hline & Error & Rate & Error & Rate & Error & Rate & Error & Rate \\
\hline$h$ & $5.1140 \mathrm{E}-02$ & $*$ & $2.2981 \mathrm{E}-03$ & $*$ & $6.3274 \mathrm{E}-02$ & $*$ & $2.4464 \mathrm{E}-03$ & $*$ \\
\hline$h / 2$ & $1.7342 \mathrm{E}-03$ & 4.8822 & $1.6195 \mathrm{E}-05$ & 7.1487 & $2.3705 \mathrm{E}-03$ & 4.7384 & $1.6012 \mathrm{E}-05$ & 7.2554 \\
\hline$h / 2^{2}$ & $9.8471 \mathrm{E}-05$ & 4.1384 & $2.2820 \mathrm{E}-07$ & 6.1491 & $1.3679 \mathrm{E}-04$ & 4.1152 & $2.2322 \mathrm{E}-07$ & 6.1645 \\
\hline$h / 2^{3}$ & $6.0027 \mathrm{E}-06$ & 4.0360 & $3.4719 \mathrm{E}-09$ & 6.0385 & $8.3830 \mathrm{E}-06$ & 4.0284 & $3.3827 \mathrm{E}-09$ & 6.0442 \\
\hline
\end{tabular}

*No data

where $D_{2}^{x}=\mathcal{F}_{M_{x}}^{-1} \Lambda_{2}^{x} \mathcal{F}_{M_{x}}$ and $D_{2}^{y}=\mathcal{F}_{M_{y}}^{-1} \Lambda_{2}^{y} \mathcal{F}_{M_{y}}$ are the second-order Fourier spectral matrices (see, e.g. [13]) in the $x$ direction and $y$ direction, respectively. The discrete energy is given by

$$
H(U(t), \dot{U}(t))=\|\dot{U}(t)\|_{2}^{2}+|U(t)|_{1}^{2}+\Delta x \Delta y \sum_{l=1}^{M_{x}} \sum_{j=1}^{M_{y}}\left(1-\cos \left(U_{l j}(t)\right)\right),
$$

where $\Delta x=2 / M_{x}$ and $\Delta y=2 / M_{y}$ are the spatial stepsizes. More details can be found in $[13,26,27]$. For this problem, we take the dimensionless parameter $\kappa=1 / 20$ and set the mesh sizes $M_{x}=M_{y}=256$. In Fig. 3 and 4, we present the simulation results and the corresponding contour plots at the time points $t=0,20,40,50,80$ and 100 with $h=0.01$. The phenomena is termed circular ring solitons and obviously is periodicity. Figure 3 demonstrates the phenomena of the periodic oscillations and radiations of the circular ring solitons as time goes on. The CPU time required to reach $T=100$ is 439.476393 seconds. The numerical data in Table 5 clearly indicate that the convergence order of the derived time integrators, which are consistent with our theoretical analysis results in Theorem 3.4. Figure 5 demonstrates that the time integrators "GTC2s4", "GTC3s6", "LTC3s4" and "LTC4s6" have better long-term computational behaviour than the chosen classical integrators. Table 6 shows the numerical precision of the long-term preservation of the discrete energy (4.4). The numerical results in Table 6 confirm the theoretical result in Theorem 3.5 .

Problem 4.3 We consider the Duffing equation (see, e.g. [32])

$$
\left\{\begin{array}{l}
\ddot{q}+\omega^{2} q=k^{2}\left(2 q^{3}-q\right), \\
q(0)=0, \quad \dot{q}(0)=\omega
\end{array}\right.
$$

with $0 \leq k<\omega$. This is a Hamiltonian system with the Hamiltonian

$$
H(p, q)=\frac{1}{2} p^{2}+\frac{1}{2} \omega^{2} q^{2}+\frac{k^{2}}{2}\left(q^{2}-q^{4}\right) .
$$

The analytic solution is given by 


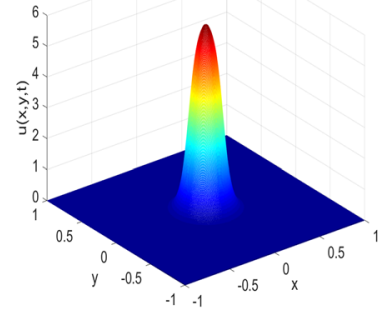

(a) $\mathrm{T}=0$

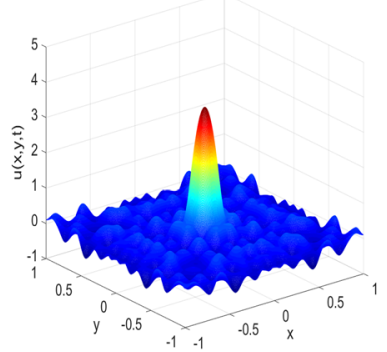

(d) $\mathrm{T}=50$

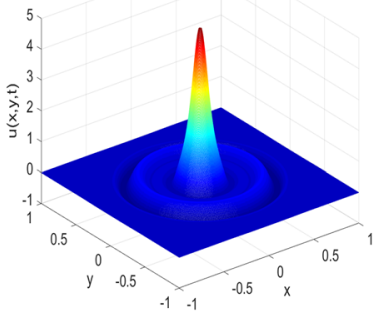

(b) $\mathrm{T}=20$

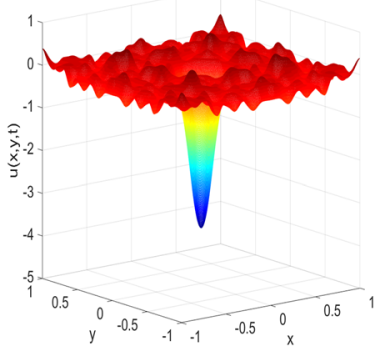

(e) $\mathrm{T}=80$

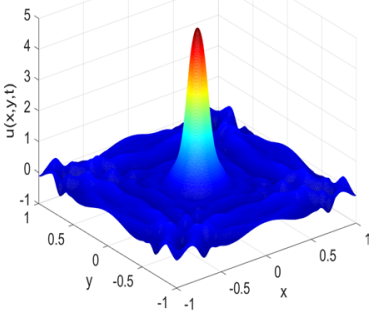

(c) $\mathrm{T}=40$

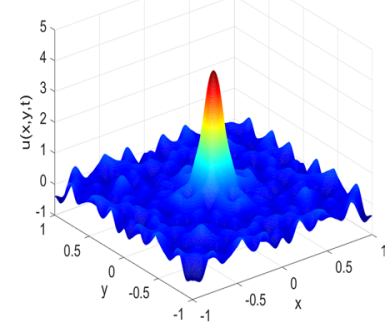

(f) $\mathrm{T}=100$

Fig. 3 Mesh graphs of numerical solutions for Problem 4.2 obtained by coupling "GTC3s6" with the two dimensional Fourier pseudo-spectral method/at the time points $t=0,20,40,50,80$ and 100.

$$
q(t)=\operatorname{sn}(\omega t, k / \omega),
$$

where $s n$ means the Jacobian elliptic function. We choose $k=0.03$ and different frequencies $\omega=10$ and 20 which are similar to those in [32]. The problem is investigated over the interval $[0,1000]$ to verify the convergence order and the precision of the energy conservation for the constructed time integrators with different $\omega$.

The data in Tables 7 and 8 show the convergence order of the time integrators "GTC2s4", "GTC3s6", "LTC3s4" and "LTC4s6" with different $\omega$. The tabular data implies that the continuous trigonometric collocation polynomial approximations with Gauss points and Lobatto points could achieve $2 s$ th order and $(2 s-2)$ th order, respectively. The logarithm of the global errors $G E=\left\|q_{N}-q(1000)\right\|_{2}$ against different steps for Problem 4.3 are plotted in Figs. 6 and 7. In comparison with the "GAS2s4", "GAS3s6", "LIIIA3s4", "LIIIA4s6", "BH1" and "BH2" integrators, the proposed time integrators in this paper have much better accuracy and cost less CPU time. However, the proposed integrators and the chosen ERKN integrators have similar precision under the same CPU time. The numerical results in Tables 9 and 10 indicate the precision of the energy conservation.

In conclusion, the numerical results for Problem 4.1, Problem 4.2 and Problem 4.3 are consistent with our theoretical analysis results. The continuous collocation polynomial approximations can be superconvergence and have better numerical behaviour 


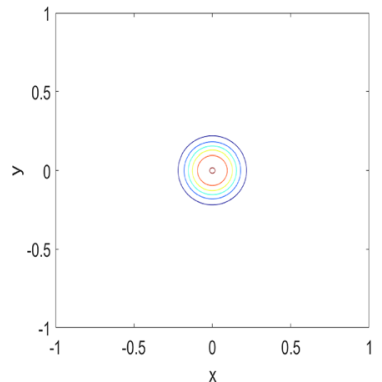

(a) $\mathrm{T}=0$

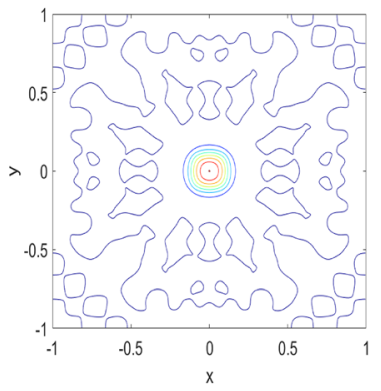

(d) $\mathrm{T}=50$

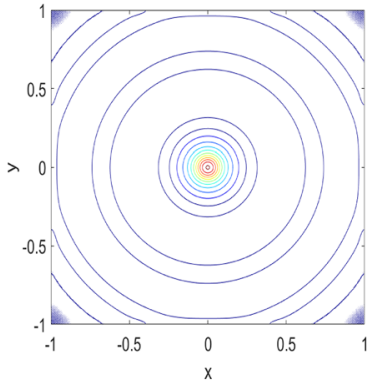

(b) $\mathrm{T}=20$

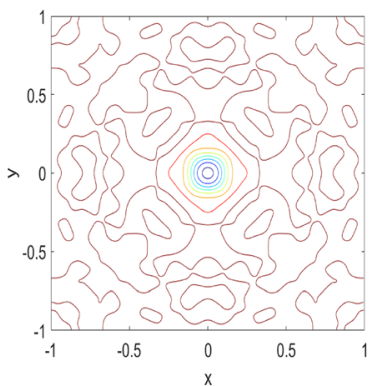

(e) $\mathrm{T}=80$

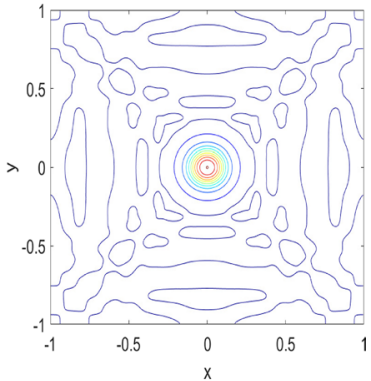

(c) $\mathrm{T}=40$

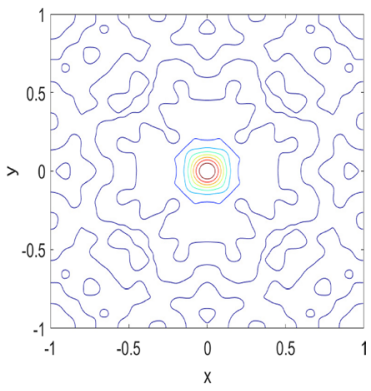

(f) $\mathrm{T}=100$

Fig. 4 Contours of numerical solutions for Problem 4.2 obtained by coupling "GTC3s6" with the two dimensional Fourier pseudo-spectral method/at the time points $t=0,20,40,50,80$ and 100.

Table 5 Temporal precision of "GTC2s4", "GTC3s6", "LTC3s4" and "LTC4s6" for solving Problem 4.2 with $h=0.08$

\begin{tabular}{|c|c|c|c|c|c|c|c|c|}
\hline \multirow[t]{2}{*}{$h$} & \multicolumn{2}{|l|}{ GTC2s4 } & \multicolumn{2}{|l|}{ GTC3s6 } & \multicolumn{2}{|l|}{ LTC3s4 } & \multicolumn{2}{|l|}{ LTC4s6 } \\
\hline & Error & Rate & Error & Rate & Error & Rate & Error & Rate \\
\hline & $8.2434 \mathrm{E}-05$ & * & $2.6349 \mathrm{E}-06$ & * & $1.2226 \mathrm{E}-04$ & * & $3.4091 \mathrm{E}-06$ & * \\
\hline$h / 2$ & $4.9537 \mathrm{E}-06$ & 4.0567 & $4.9539 \mathrm{E}-08$ & 5.7331 & $7.5801 \mathrm{E}-06$ & 4.0116 & $6.5801 \mathrm{E}-08$ & 5.6951 \\
\hline$h / 2^{2}$ & $3.0966 \mathrm{E}-07$ & 3.9997 & $8.1146 \mathrm{E}-10$ & 5.9319 & $4.7386 \mathrm{E}-07$ & 3.9997 & $1.0826 \mathrm{E}-09$ & 5.9255 \\
\hline$h / 2^{3}$ & $1.9359 \mathrm{E}-08$ & 3.9996 & $1.2790 \mathrm{E}-11$ & 5.9875 & $2.9621 \mathrm{E}-08$ & 3.9997 & $1.7079 \mathrm{E}-11$ & 5.9862 \\
\hline
\end{tabular}

*No data

than the chosen classical integrators. Comparing with the classical implicit methods, i.e., "GAS2s4" method, "GAS3s6" method, "LIIIA3s4" method, "LIIIA4s6" method, "BH1" method and "BH2" method, the proposed time integrators "GTC2s4", "GTC3s6", "LTC3s4" and "LTC4s6" have better precision while cost less CPU time. However, comparing with the explicit ERKN3s4 method and ERKN7s6 method, our proposed time integrators have similar precision under the same CPU time. Moreover, the numerical results in [39] indicated that the ERKN3s4 and ERKN7s6 methods have 

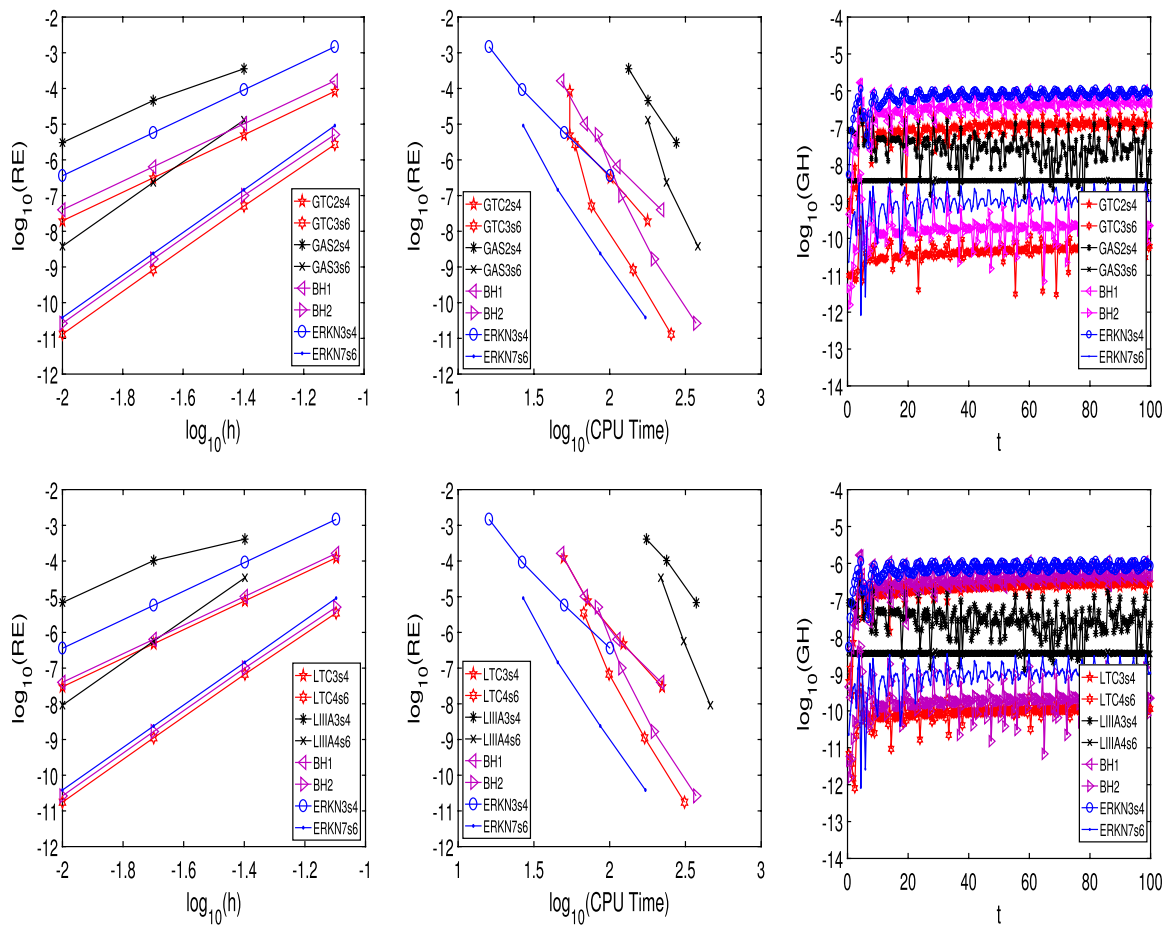

Fig. 5 Results for Problem 4.2. (Left) the $\log -\log$ plot of posterior error $R E$ against different time stepsizes $h$. (Middle) the log-log plot of posterior error $R E$ against CPU time. (Right) the logarithm of the discrete energy error $G H$ against $t$

Table 6 Numerical precision of the preservation of the discrete energy for Problem 4.2 with $h=0.08$

\begin{tabular}{|c|c|c|c|c|c|c|c|c|}
\hline \multirow[t]{2}{*}{$h$} & \multicolumn{2}{|l|}{ GTC2s4 } & \multicolumn{2}{|l|}{ GTC3s6 } & \multicolumn{2}{|l|}{ LTC3s4 } & \multicolumn{2}{|l|}{ LTC4s6 } \\
\hline & Error & Rate & Error & Rate & Error & Rate & Error & Rate \\
\hline$h$ & $1.8869 \mathrm{E}-06$ & $*$ & $6.3650 \mathrm{E}-09$ & $*$ & $3.1193 \mathrm{E}-06$ & $*$ & $9.0491 \mathrm{E}-09$ & $*$ \\
\hline$h / 2$ & $1.1854 \mathrm{E}-07$ & 3.9926 & $9.1859 \mathrm{E}-11$ & 6.1146 & $1.9403 \mathrm{E}-07$ & 4.0069 & $1.3681 \mathrm{E}-10$ & 6.0475 \\
\hline$h / 2^{2}$ & $7.5259 \mathrm{E}-09$ & 3.9773 & $1.4297 \mathrm{E}-12$ & 6.0056 & $1.2354 \mathrm{E}-08$ & 3.9733 & $2.1194 \mathrm{E}-12$ & 6.0124 \\
\hline$h / 2^{3}$ & $4.6940 \mathrm{E}-10$ & 4.0030 & $4.2044 \mathrm{E}-13$ & - & $7.7083 E-10$ & 4.0024 & $4.1939 \mathrm{E}-13$ & - \\
\hline
\end{tabular}

*No data

Table 7 Temporal precision of "GTC2s4", "GTC3s6", "LTC3s4" and "LTC4s6" for solving Problem 4.3 with $h=0.2$ and $\omega=10$

\begin{tabular}{|c|c|c|c|c|c|c|c|c|}
\hline \multirow[t]{2}{*}{$h$} & \multicolumn{2}{|l|}{ GTC2s4 } & \multicolumn{2}{|l|}{ GTC3s6 } & \multicolumn{2}{|l|}{ LTC3s4 } & \multicolumn{2}{|l|}{ LTC4s6 } \\
\hline & Error & Rate & Error & Rate & rror & Rate & Error & Rate \\
\hline$h$ & $2.2948 E-04$ & $*$ & $6.5535 \mathrm{E}-06$ & $*$ & 3.374 & $*$ & $8.7509 E-06$ & $*$ \\
\hline$h / 2$ & $1.5263 \mathrm{E}-05$ & 3.9102 & $1.0957 \mathrm{E}-07$ & 5.9024 & $2.2811 \mathrm{E}-05$ & 3.8868 & $1.4485 \mathrm{E}-07$ & 5.9168 \\
\hline$h / 2^{2}$ & $9.6938 \mathrm{E}-07$ & 3.9768 & $1.7381 \mathrm{E}-09$ & 5.9782 & $1.4532 \mathrm{E}-06$ & 3.9724 & $2.3046 \mathrm{E}-09$ & 5.9739 \\
\hline$h / 2^{3}$ & $6.0899 \mathrm{E}-08$ & 3.9926 & $2.8857 \mathrm{E}-11$ & 5.9124 & $9.1311 \mathrm{E}-08$ & 3.9923 & $3.7772 \mathrm{E}-11$ & 5.9311 \\
\hline
\end{tabular}

*No data 

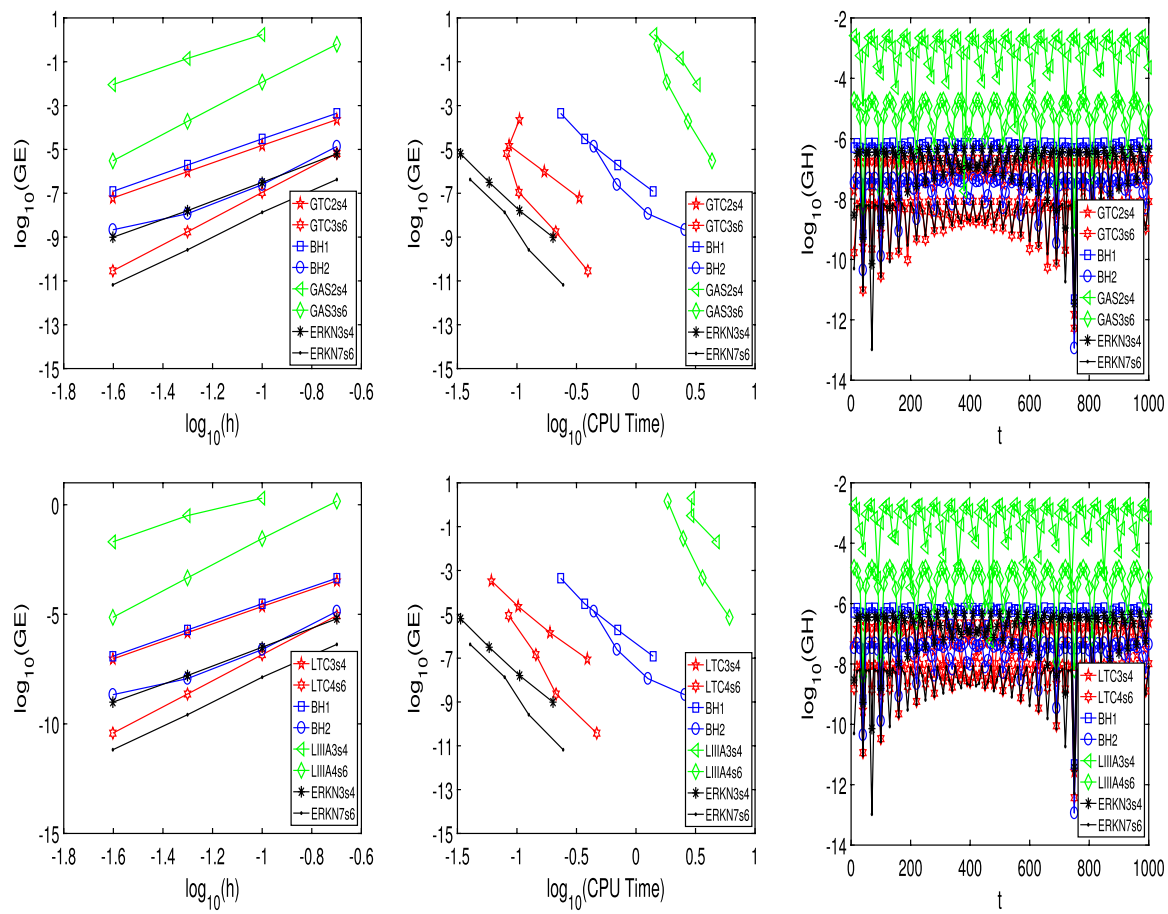

Fig. 6 Results for Problem 4.3 with $\omega=10$. (Left) the $\log -\log$ plot of global error $G E$ against different time stepsizes $h$. (Middle) the $\log -\log$ plot of global error $R E$ against CPU time. (Right) the logarithm of the global energy error $G H$ against $t$

Table 8 Temporal precision of "GTC2s4", "GTC3s6", "LTC3s4" and "LTC4s6" for solving Problem 4.3 with $h=0.1$ and $\omega=20$

\begin{tabular}{|c|c|c|c|c|c|c|c|c|}
\hline \multirow[t]{2}{*}{$h$} & \multicolumn{2}{|l|}{ GTC2s4 } & \multicolumn{2}{|l|}{ GTC3s6 } & \multicolumn{2}{|l|}{ LTC3s4 } & \multicolumn{2}{|l|}{ LTC4s6 } \\
\hline & Error & Rate & Error & Rate & Error & Rate & Error & Rate \\
\hline$h$ & $1.1468 \mathrm{E}-04$ & $*$ & $3.2996 \mathrm{E}-06$ & $*$ & $1.6896 \mathrm{E}-04$ & * & $4.3554 \mathrm{E}-06$ & * \\
\hline$h / 2$ & $7.6411 \mathrm{E}-06$ & 3.9077 & $5.4632 \mathrm{E}-08$ & 5.9164 & $1.1406 \mathrm{E}-05$ & 3.8888 & $7.2744 \mathrm{E}-08$ & 5.9038 \\
\hline$h / 2^{2}$ & $4.8518 \mathrm{E}-07$ & 3.9772 & $8.6855 \mathrm{E}-10$ & 5.9750 & $7.2682 \mathrm{E}-07$ & 3.9721 & $1.1541 \mathrm{E}-09$ & 5.9779 \\
\hline$h / 2^{3}$ & $3.0467 \mathrm{E}-08$ & 3.9932 & $1.5864 \mathrm{E}-11$ & 5.7748 & $4.5693 \mathrm{E}-08$ & 3.9916 & $2.0141 \mathrm{E}-11$ & 5.8405 \\
\hline
\end{tabular}

*No data

better numerical behaviour than the ARKN3s4, TFCr2 and TFCr3 methods. Therefore, we could confirm that the proposed time integrators in this paper would be better than the ARKN3s4, TFCr2 and TFCr3 methods. The Gautschi-type methods are time integrators with second order precision at most. We will not compare with the Gautschitype, ARKN and TFC methods in this paper. 

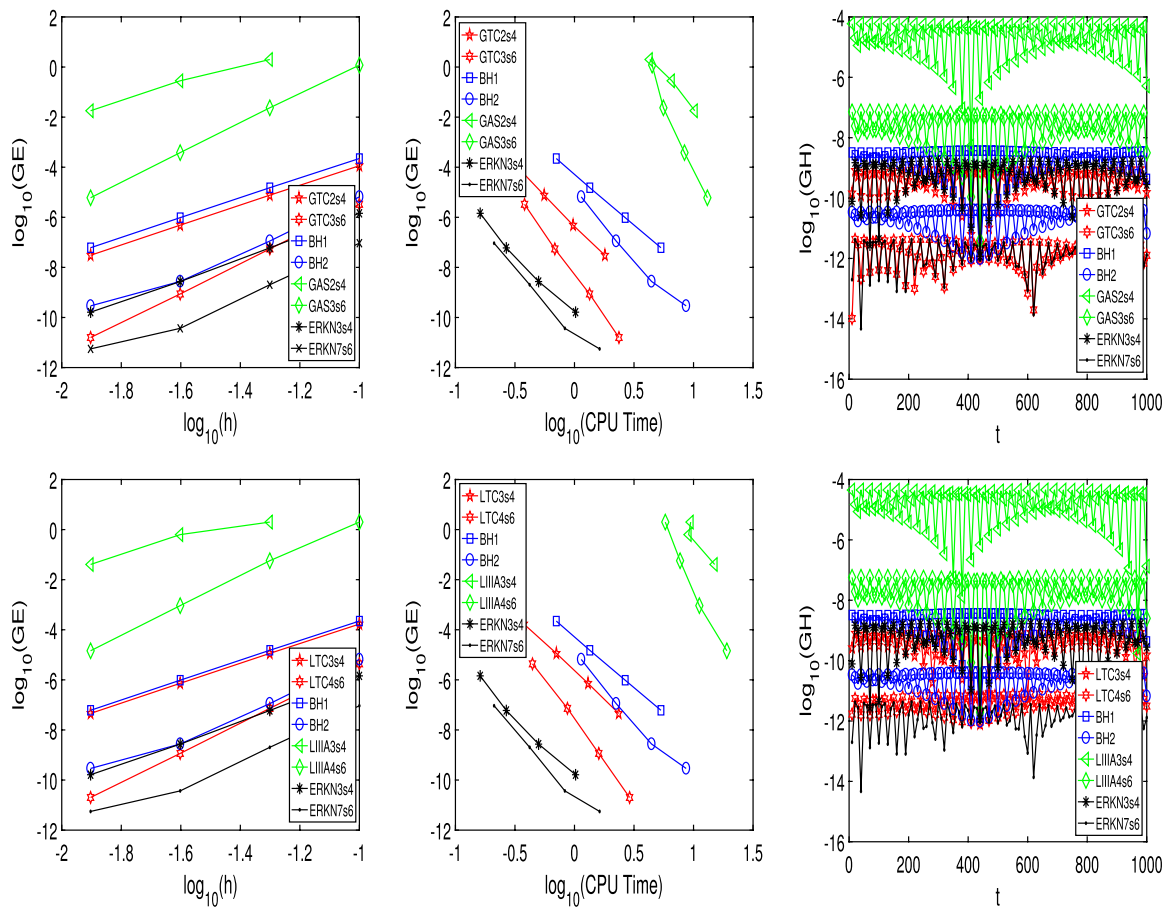

Fig. 7 Results for problem 4.3 with $\omega=20$. (Left) the log-log plot of global error $G E$ against different time stepsizes $h$. (Middle) the log-log plot of global error $R E$ against CPU time. (Right) the logarithm of the global energy error $G H$ against $t$

Table 9 Numerical precision of the preservation of the global energy for Problem 4.3 with $h=0.2$ and $\omega=10$

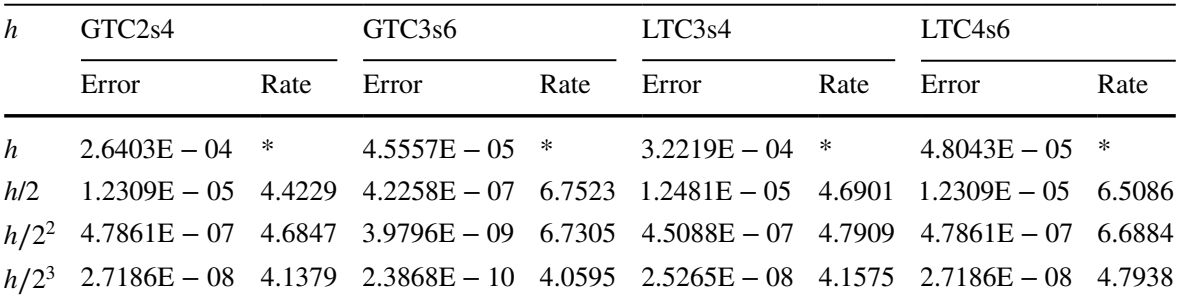

*No data

Table 10 Numerical precision of the preservation of the global energy for Problem 4.3 with $h=0.1$ and $\omega=20$

\begin{tabular}{|c|c|c|c|c|c|c|c|c|}
\hline \multirow[t]{2}{*}{$h$} & \multicolumn{2}{|l|}{ GTC2s 4} & \multicolumn{2}{|l|}{ GTC3s6 } & \multicolumn{2}{|l|}{ LTC3s4 } & \multicolumn{2}{|l|}{ LTC4s6 } \\
\hline & Error & Rate & Error & Rate & Error & Rate & Error & Rate \\
\hline$h$ & $2.6403 E-04$ & $*$ & $4.5556 \mathrm{E}-05$ & $*$ & $3.2219 \mathrm{E}-04$ & $*$ & $4.8043 \mathrm{E}-05$ & $*$ \\
\hline$h / 2$ & $1.2309 \mathrm{E}-05$ & 4.4229 & $4.2276 \mathrm{E}-07$ & 6.7517 & $1.2481 \mathrm{E}-05$ & 4.6901 & $5.2763 \mathrm{E}-07$ & 6.5087 \\
\hline$h / 2^{2}$ & $4.7861 \mathrm{E}-07$ & 4.6847 & $4.6871 \mathrm{E}-09$ & 6.4950 & $4.5159 \mathrm{E}-07$ & 4.7886 & $5.1139 \mathrm{E}-09$ & 6.6889 \\
\hline$h / 2^{3}$ & $2.7185 \mathrm{E}-08$ & 4.1380 & $1.5256 \mathrm{E}-09$ & - & $2.6544 \mathrm{E}-08$ & 4.0886 & $1.4599 \mathrm{E}-09$ & - \\
\hline
\end{tabular}

*No data 


\section{Conclusion}

Taking into account the superiority of the continuous collocation methods and the trigonometric integrators, we proposed and analysed the continuous trigonometric collocation polynomial approximations for the highly oscillatory hyperbolic system (1.1) in this paper. The derived trigonometric collocation integrators inherit the superconvergence of the classical continuous collocation methods and also can preserve the oscillatory structure of the underlying highly oscillatory systems. The resulting trigonometric collocation integrators were analysed in details for the local error bounds, long-term convergence, superconvergence, symmetry and long-term energy conservation. Furthermore, the remarkable numerical behaviour of the continuous trigonometric collocation polynomial approximations was demonstrated by the numerical experiments in comparison with the existing numerical methods in the literature.

Acknowledgments The research is supported in part by the Natural Science Foundation of China under Grant 11801280 and 12071419 , by the Natural Science Foundation of the Jiangsu Higher Education Institutions under Grant 18KJB110020, by the Natural Science Foundation of Jiangsu Province under Grant BK20180780, and by the Scientific Research Foundation of Nanjing University of Information Science \& Technology under Grant 2017r072.

Open Access This article is licensed under a Creative Commons Attribution 4.0 International License, which permits use, sharing, adaptation, distribution and reproduction in any medium or format, as long as you give appropriate credit to the original author(s) and the source, provide a link to the Creative Commons licence, and indicate if changes were made. The images or other third party material in this article are included in the article's Creative Commons licence, unless indicated otherwise in a credit line to the material. If material is not included in the article's Creative Commons licence and your intended use is not permitted by statutory regulation or exceeds the permitted use, you will need to obtain permission directly from the copyright holder. To view a copy of this licence, visit http://creativecommons.org/licen ses/by/4.0/.

\section{References}

1. Abramowitz, M., Stegun, I.: Handbook of Mathematical Functions with Formulas, Graphs, and Mathematical Tables. Dover publications, New York (1964)

2. Bao, W.Z., Dong, X.C.: Analysis and comparison of numerical methods for the Klein-Gordon equation in the nonrelativistic limit regime. Numer. Math. 120, 189-229 (2012)

3. Bank, R., Graham, R.L., Stoer, J., Varga, R., Yserentant, H.: High order difference method for time dependent PDE. Springer, Berlin, Heidelberg (2008)

4. Bátkai, A., Farkas, B., Csomós, P., Ostermann, A.: Operator semigroups for numerical analysis. 15th Internet Seminar, p. 12 (2011)

5. Davydov, A.S.: Quantum Mechanics, 2nd edn. Pergamon, Oxford (1976)

6. Fang, Y.L., Liu, C.Y., Wang, B.: Efficient energy-preserving methods for general nonlinear oscillatory Hamiltonian system. Acta Math. Sin. English Ser. 34, 1864-1878 (2018)

7. Franco, J.M.: New methods for oscillatory systems based on ARKN methods. Appl. Numer. Math. 56, 1040-1053 (2006)

8. García-Archilla, B., Sanz-Serna, J.M., Skeel, R.D.: Long-time-step methods for oscillatory differential equations. SIAM J. Sci. Comput. 20, 930-963 (1998)

9. Gauckler, L.: Error analysis of trigonometric integrators for semilinear wave equations. SIAM J. Numer. Anal. 53, 1082-1106 (2015) 
10. Gautschi, W.: Numerical integration of ordinary differential equations based on trigonometric polynomials. Numer. Math. 3, 381-397 (1961)

11. Glassey, R.: On the asymptotic behavior of nonlinear wave equations. Trans. Am. Math. Soc. 182, 187-200 (1973)

12. Grimm, V.: On error bounds for the Gautschi-type exponential integrator applied to oscillatory second-order differential equations. Numer. Math. 100, 71-89 (2005)

13. Gong, Y.Z., Wang, Q., Wang, Y.S., Cai, J.X.: A conservative Fourier pseudo-spectral method for the nonlinear Schrödinger equation. J. Comput. Phys. 328, 354-370 (2017)

14. Gottlieb, D., Orszag, S.: Numerical Analysis of Spectral Methods: Theory and Applications. Society for Industrial and Applied Mathematics, Philadelphia (1993)

15. Hairer, E., Lubich, C.: Long-time energy conservation of numerical methods for oscillatory differential equations. SIAM J. Numer. Anal. 38, 414-441 (2000)

16. Hairer, E., Lubich, C., Wanner, G.: Geometric Numerical Integration: Structure-Preserving Algorithms for Ordinary Differential Equations, 2nd edn. Springer, Berlin, Heidelberg (2006)

17. Hesthaven, J.S., Gottlieb, S., Gottlieb, D.: Spectral methods for time-dependent problems. Monographs on Applied and Computational Mathematics. Cambridge University Press, Cambridge (2007)

18. Hochbruck, M., Ostermann, A.: Exponential Runge-Kutta methods for parabolic problems. Appl. Numer. Math. 53, 323-339 (2005)

19. Hochbruck, M., Ostermann, A.: Exponential integrators. Acta Numer. 19, 209-286 (2010)

20. Lakestani, M., Dehghan, M.: Collocation and finite difference-collocation methods for the solution of nonlinear Klein-Gordon equation. Comput. Phys. Commun. 181, 1392-1401 (2010)

21. Li, S., Vu-Quoc, L.: Finite difference calculus invariant structure of a class of algorithms for the nonlinear Klein-Gordon equation. SIAM J. Numer. Anal. 32, 1839-1875 (1995)

22. Liu, C.Y., Iserles, A., Wu, X.: Symmetric and arbitrarily high-order Birkhoff-Hermite time integrators and their long-time behaviour for solving nonlinear Klein-Gordon equations. J. Comput. Phys. 356, 1-30 (2018)

23. Liu, C.Y., Wu, X.: Arbitrarily high-order time-stepping schemes based on the operator spectrum theory for high-dimensional nonlinear Klein-Gordon equations. J. Comput. Phys. 340, 243-275 (2017)

24. Liu, C.Y., Wu, X.: The boundness of the operator-valued functions for multidimensional nonlinear wave equations with applications. Appl. Math. Lett. 74, 60-67 (2017)

25. Liu, C.Y., Wu, X., Shi, W.: New energy-preserving algorithms for nonlinear Hamiltonian wave equation equipped with Neumann boundary conditions. Appl. Comput. Math. 339, 588-606 (2018)

26. Shen, J., Tang, T.: Spectral and High-Order Methods with Applications. Science Press, Beijing (2006)

27. Shen, J., Tang, T., Wang, L.L.: Spectral Methods: Algorithms, Analysis, Applications. Springer, Berlin (2011)

28. Shi, W., Liu, K., Wu, X.Y., Liu, C.Y.: An energy-preserving algorithm for nonlinear Hamiltonian wave equations with Neumann boundary conditions. Calcolo 54, 1379-1402 (2017)

29. Simon, J.C.H., Taflin, E.: The Cauchy problem for non-linear Klein-Gordon equations. Commun. Math. Phys. 152, 433-478 (1993)

30. Smith, G.D.: Numerical Solution of Partial Differential Equations. Oxford University Press, London (1965)

31. Thomée, V.: Galerkin Finite Element Methods for Parabolic Problems. Springer, Berlin (1997)

32. Wang, B., Iserles, A., Wu, X.: Arbitrary-order trigonometric Fourier collocation methods for multi-frequency oscillatory systems. Found. Comput. Math. 16, 151-181 (2016)

33. Wang, B., Yang, H., Meng, F.: Sixth order symplectic and symmetric explicit ERKN schemes for solving multi-frequency oscillatory nonlinear Hamiltonian equations. Calcolo 54, 117-140 (2017)

34. Wang, B., Wu, X.Y.: The formulation and analysis of energy-preserving schemes for solving highdimensional nonlinear Klein-Gordon equations. IMA J. Numer. Anal. 39, 2016-2044 (2019)

35. Wang, B., Wu, X.Y.: Global error bounds of one-stage explicit extended RKN integrators for semilinear wave equations. Numer. Algorithms 81, 1203-1218 (2019)

36. Wang, B., Wu, X.Y., Meng, F.W.: Trigonometric collocation methods based on Lagrange basis polynomials for multi-frequency oscillatory second-order differential equations. J. Comput. Appl. Math. 313, 185-201 (2017) 
37. Wang, Y., Zhao, X.F.: Symmetric high order Gautschi-type exponential wave integrators pseudospectral method for the nonlinear Klein-Gordon equation in the nonrelativistic limit regime. Int. J. Numer. Anal. Mod. 15, 405-427 (2018)

38. Wu, X.Y., Liu, K., Shi, W.: Structure-Preserving Algorithms for Oscillatory Differential Equations II. Springer, Heidelberg (2015)

39. Wu, X. Y., Wang, B., Mei, L. Oscillation-preserving algorithms for efficiently solving highly oscillatory second-order ODEs, Numer. Algor. https://doi.org/10.1007/s11075-020-00908-7

40. Wu, X.Y., Wang, B.: Recent Developments in Structure-Preserving Algorithms for Oscillatory Differential Equations. Springer, Singapore (2018)

41. Wu, X.Y., You, X., Xia, J.L.: Order conditions for ARKN methods solving oscillatory systems. Comput. Phys. Comm. 180, 2250-2257 (2009)

42. Wu, X.Y., You, X., Wang, B.: Structure-Preserving Algorithms for Oscillatory Differential Equations. Springer, Heidelberg (2013)

Publisher's Note Springer Nature remains neutral with regard to jurisdictional claims in published maps and institutional affiliations. 